\title{
Recognition determinants of broadly neutralizing human antibodies against dengue viruses
}

\begin{abstract}
Alexander Rouvinski1,2*, Pablo Guardado-Calvo ${ }^{1,2^{*}}$, Giovanna Barba-Spaeth ${ }^{1,2^{*}}$, Stéphane Duquerroy ${ }^{1,2,3}$, Marie-Christine Vaney1,2, Carlos M. Kikuti1,2‡, M. Erika Navarro Sanchez ${ }^{1,2 \ddagger}{ }^{1}$, Wanwisa Dejnirattisai4, Wiyada Wongwiwat ${ }^{4}$, Ahmed Haouz ${ }^{5}$, Christine Girard-Blanc ${ }^{5}$, Stéphane Petres ${ }^{5}$, William E. Shepard6, Philippe Desprès ${ }^{7 \ddagger}$, Fernando Arenzana-Seisdedos ${ }^{8}$, Philippe Dussart ${ }^{\ddagger \ddagger}$, Juthathip Mongkolsapaya ${ }^{4,10 \S}$, Gavin R. Screaton ${ }^{4}$ and Félix A. Rey ${ }^{1,2,5 \S}$
\end{abstract}

1Institut Pasteur, Département de Virologie, Unité de Virologie Structurale, 75724 Paris Cedex 15, France ${ }^{2}$ CNRS UMR 3569 Virologie, 75724 Paris Cedex 15, France

3Université Paris-Sud, Faculté des Sciences, 91405 Orsay, France

${ }^{4}$ Division of Immunology and Inflammation, Department of Medicine, Hammersmith Hospital Campus, Imperial College London, UK

5Institut Pasteur, Protéopôle, CNRS UMR 3528, 75724 Paris Cedex 15, France

${ }^{6}$ Synchrotron SOLEIL, L’Orme des Merisiers, Saint Aubin, BP48, 91192 Gif-sur-Yvette, France

7Institut Pasteur, Département de Virologie, Unité des Interactions Moléculaires Flavivirus-Hôtes, 75724

Paris Cedex 15, France

8Institut Pasteur, Département de Virologie, Unité de Pathogénie Virale, INSERM U1108, 75724 Paris

Cedex 15, France

${ }^{9}$ Institut Pasteur de Guyane, BP 6010, 97306 Cayenne, French Guiana

10Dengue Hemorrhagic Fever Research Unit, Office for Research and Development, Faculty of Medicine, Siriraj Hospital, Mahidol University, Bangkok, Thailand

${ }^{*}$ These authors contributed equally to this work

‡Present addresses: $\quad$ Institut Curie, 75248 Paris Cedex 05, France (CMK);

Altravax, Inc. 725 San Aleso Avenue, Suite 2, Sunnyvale, CA 94085, USA (MENS); U1157 INSERM GIP-CYROI, 97491 Saint Clotilde, La Réunion, France (PDe); Institut Pasteur du Cambodge, 5 Monivong blvd, PO Box 983, Phnom Penh, Cambodia (PDu)

Running title: X-ray structures of the dengue virus E protein in complex with human antibodies Manuscript count: 2842 word (excluding summary and title page)

$\S$ Correspondence:

rey@pasteur.fr (FAR);

g.screaton@imperial.ac.uk (GRS);

j.mongkolsapaya@imperial.ac.uk (JM) 
Dengue disease is caused by four different flavivirus ${ }^{1}$ serotypes, which infect 390 million people yearly with $25 \%$ symptomatic cases ${ }^{2}$ and for which no licensed vaccine is available. Recent phase III vaccine trials showed partial protection, and in particular no protection for dengue virus serotype 2 (DENV$2)^{3,4}$. Structural studies so far have characterized only epitopes recognized by serotype specific human antibodies ${ }^{5,6}$. We recently isolated human antibodies potently neutralizing all four DENV serotypes ${ }^{7}$. Here we describe the X-ray structures of four of these broadly neutralizing antibodies (bnAbs) in complex with the envelope glycoprotein E from DENV-2, revealing that the recognition determinants are at a serotype conserved site at the $\mathrm{E}$ dimer interface, including the exposed main chain of the $\mathrm{E}$ fusion $\operatorname{loop}^{8}$ and the two conserved glycan chains. This "E-dimer dependent epitope" (EDE) is also the binding site for the viral glycoprotein prM during virus maturation in the secretory pathway of the infected cell ${ }^{9}$, explaining its conservation across serotypes and highlighting an Achilles heel of the virus with respect to antibody neutralization. These findings will be instrumental for devising novel immunogens to protect simultaneously against all four serotypes of dengue virus.

Exposed at the surface of infectious mature DENV particles, protein E is the sole target of neutralizing antibodies. It displays an icosahedral arrangement in which $90 \mathrm{E}$ dimers completely coat the viral surface ${ }^{10,11}$ and which is sensitive to the environmental pH. Upon entry of DENV into cells via receptor-mediated endocytosis, the acidic 
endosomal environment triggers an irreversible fusogenic conformational change in E that leads to fusion of viral and endosomal membranes ${ }^{1}$. The structure of the isolated $\mathrm{E}$ dimer has been determined by X-ray crystallography using the soluble ectodomain $(\mathrm{sE})^{8,12}$. Protein E is relatively conserved, displaying about $65 \%$ amino acid sequence identity when comparing the most distant DENV serotypes. In particular, there are two conserved N-linked glycosylation sites at positions N67 and N153. To examine its interaction with the antibodies, we selected four highly potent bnAbs identified in the accompanying work: 747(4) A11 and 747 B7 (EDE2 group, requiring glycosylation at position N153 for efficient binding) and 752-2 C8 and 753(3) C10 (EDE1 group, binding regardless of the glycosylation at $\mathrm{N} 153)^{7}$ - referred to as $\mathrm{A} 11, \mathrm{~B} 7, \mathrm{C} 8$ and $\mathrm{C} 10$ from hereon. The EDE2 bnAbs were isolated from the same patient (who had a secondary infection with DENV-2), and are somatic variants of the same IgG clone, derived from the IGHV3-74 and IGLV2-23 germ lines. The heavy chain has a very long (26 amino acids, IMGT convention) complementarity-determining region 3 (CDR H3). The EDE1 bnAbs were isolated from different patients and derive from VH and VL genes IGHV3-64 and IGKV3-11, (EDE1 C8, the patient appeared to have a primary infection of undetermined serotype) and IGHV1-3* and IGLV2-14 (EDE1 C10, from a patient with secondary DENV1 infection). The analysis of the genes coding for these antibodies is summarized in Table 1.

Recombinant sE is mainly monomeric in solution, but crystallizes as dimer 8,12,13. The crystal structures of the antibody/antigen complexes were determined as described in the Methods section, to a resolution between $3.0 \AA$ and $3.2 \AA$ for the 
complexes with B7, C10 and C8; and 3.85Å for the complex with A11 (ED Table 1). They show that all four bnAbs bind in a similar way (Fig. 1), interacting with both subunits and leaving a similar footprint on the sE dimer (ED Figure 1).

The antibody/antigen contacts are centered in a valley lined by the $b$ strand on the domain II side, and by the "150 loop" of domain I of the adjacent subunit on the opposite side (Fig 1d), with the heavy chain contacting both N67 and N153 glycans across the E dimer interface (Fig 1e-g). The 150 loop spans residues 148-159, connecting $\beta$-strands $\mathrm{E}_{0}$ and $\mathrm{F}_{0}$ of domain $\mathrm{I}^{14}$, and carries the $\mathrm{N} 153$ glycan. The total buried surface area (BSA) per epitope ranges between $1050 \AA^{2}$ and $1400 \AA^{2}$, and the surface complementarity coefficient ${ }^{15}$ is between 0.67 and 0.74 (ED Table 2), which are values typical for antibody/antigen complexes. The surface electrostatic potentials of epitope and paratopes are mildly charged (ED Fig. 2), with a relatively complementary charge distribution. The EDE2 antibodies recognize sE essentially via the heavy chain, with the light chain involved only in contacts with the N153 glycan. The heavy chain buries nearly $85 \%$ of the total BSA - $63 \%$ belonging to the long EDE2 CDR H3, which forms a protrusion matching the concave surface of the $\mathrm{sE}$ dimer. This protrusion is preformed in the antibody, as shown by the $1.7 \AA$ resolution structure of the unliganded EDE2 A11 scFv (ED Fig. 3), indicating no entropic cost for binding. In contrast, the EDE1 antibodies engage a substantial amount of light chain contacts from all 3 CDR and framework (FRW) regions, contributing in total 40\% in C8 and 47\% in C10 of the BSA in the complex with sE. Although the germlines are different between EDE1 C8 and C10 and the CDRs contribute differently to the paratope (Fig. 2a), the chemical nature of the 
residues involved is similar, including a clear clustering of aromatic side chains - a feature shared with the EDE2 bnAbs (Fig. 2b).

The bnAb contacts cluster on residues at the dimer interface (Fig. 1c) that are conserved across the four serotypes (ED Fig. 4a), explaining their cross-reactivity. On domain II, both EDE1 and EDE2 bnAbs target the same residues (ED Figs. 4 and 5), which map to three main polypeptide segments: the $b$ strand (aa 67-74, bearing the N67 glycan), the fusion loop and residues immediately upstream (aa 97-106), and the ij loop (aa 246-249). In contrast, on the opposite subunit the residues targeted are different: the EDE2 bnAbs interact with the 150 loop and the N153 glycan chain, whereas EDE1 bnAbs target domain I and III and induce disorder of the 150 loop. The structure of unliganded DENV-2 sE of the same strain (FGA-02, Fig. 1b-d), determined in parallel, was useful in assessing that EDE1 antibody binding indeed displaces the 150 loop. This is relevant because a previous structure of DENV-4 $\mathrm{sE}$ in complex with an antibody that binds away from the EDE also had the 150 loop disordered ${ }^{13}$, highlighting an intrinsic mobility in this area depending on the E amino acid sequence. Displacement of the 150 loop allows the EDE1 light chain to come closer to $\mathrm{sE}$ and interact with domain III (compare panel e with f and g in Fig.1) in the region of the "A strand" epitope, which has been structurally characterized previously for murine DENV cross-reactive antibodies $^{16,17}$. These domain III contacts are centered on the conserved E residue K310, the side chain of which makes a lid covering the indole ring of W101 of the fusion loop (ED Fig. 6a), in an important stabilizing E dimer contact. 
The bnAbs make extensive interactions with the glycan chains, both at positions N67 and N153 of E (ED Figs. 6 and 7), using opposite sides of the paratope (Fig. 2a). sE in complex with EDE1 C8 displays the highest ordered N67 glycan structure (ED Fig. 7), with interactions with CDR H2 (Fig. 2a). The distant mannose residues contact the framework region 3 (FRW H3, ED Figs. 4b and 7). Except for EDE1 C10 (which is very close to its germ line, Table 1), several FRW H3 residues in the other bnAbs analyzed have undergone changes (ED Fig. 4b), suggesting affinity maturation to recognize the sugars. With respect to the N153 glycan, the electron density is clear for its core 6 sugar residues in the structure of sE in complex with the EDE2 bnAbs (including in omit maps, as shown in ED Fig. 7). A short $\alpha$-helix in A11 and B7 CDR H3 projects aromatic side chains that pack against the sugar residues 1, 3 and 4 of the N153 glycan. The most distant residues of the glycan, mannoses 4, 5 and 6, are in contact with the light chain, via residues from CDR L2, including several hydrogen bonds (ED Fig. 7 and SI)

The glycine rich fusion loop in the E dimer is such that it essentially exposes main chain atoms, while the hydrophobic side chains are mostly buried. Together with the main chain of the $i j$ loop, main chain atoms make a large surface patch that is augmented by the exposed edge of the $b$ strand (Fig. 3). The bnAbs make a number of specific hydrogen bonds to the sE main chain, (ED Fig. 6, see also list of interactions in SI). Furthermore, the exposed side chains in this area are mostly conserved, resulting in a core region of the EDE that is serotype invariant, with non-conserved residues essentially at the periphery. The principal binding determinants of the EDE bnAbs thus appear to be the conformation of the main chain of the fusion loop and its immediate 
neighbors in the context of an intact $\mathrm{E}$ dimer. This contrasts with the other major class of antibodies isolated from humans, which recognize the fusion loop sequence in a context independent of the quaternary organization ${ }^{7}$. The latter are cross reactive but poorly neutralizing and have a strong infection enhancing potential ${ }^{18}$.

What is the reason for the strong conservation of this site across serotypes? In the infected cell, newly synthesized immature DENV virions bud into the ER lumen, where the $\mathrm{pH}$ is neutral. These particles contain 180 copies of a heterodimer of $\mathrm{E}$ with the precursor membrane glycoprotein prM at their surface ${ }^{19,20}$. As they are subsequently transported to the external medium across the Golgi apparatus, where the $\mathrm{pH}$ is acidic, the interaction with prM protects $\mathrm{E}$ from undergoing a premature acidinduced fusogenic conformational change ${ }^{1}$. The E/prM heterodimers reversibly associate as 60 trimers or 90 dimers at neutral or acidic $\mathrm{pH}$, respectively,21. Upon cleavage of its viral membrane tether by the TGN resident furin protease, prM remains bound to E dimers as long as the environment is acidic but it is released when the particle reaches the exterior of the cell (where the $\mathrm{pH}$ is neutral), thereby activating the virion to become fusogenic upon re-encountering the acidic endosomal environment of a new cell. The binding site of prM on the E dimer ${ }^{9,21}$ maps precisely to the EDE (Fig. 3), explaining its high conservation. As the E dimer conformation with bound prM was observed on immature particles at low $\mathrm{pH}$ only ${ }^{9}$, at neutral $\mathrm{pH}$ the EDE is not formed, and accordingly bnAbs do not bind to particles that are $100 \%$ immature ${ }^{7}$, which are formed exclusively of $\mathrm{E} / \mathrm{prM}$ trimers ${ }^{19}$. But the efficient binding of the bnAbs to particles that have more than $60 \%$ uncleaved $\operatorname{prM}^{7}$, which have been shown to display 
patches of immature E/prM trimers at their surface 22,23 , shows that these trimers must undergo a dynamic exchange with dimers. As the bnAbs have a higher affinity they outcompete uncleaved prM on partially immature virions from its interaction with E dimers, binding by conformational selection ${ }^{24}$. This process effectively displaces the equilibrium toward dimers, similar to the bnAb-induced shift toward dimers of the sE monomer-dimer equilibrium in solution (data not shown). Because the degree of prM cleavage is variable and depends on the particular cell in which the virus was replicated, the fact that these bnAbs bind efficiently partially mature particles is expected to be important for protection in humans.

The EDE is totally circumscribed to the E dimer and therefore it does not depend on the higher order arrangement of dimers, as recently suggested for other quaternary epitopes on the DENV particle ${ }^{25}$ based on studies on a different flavivirus, the West Nile virus $^{26}$. Recent studies on DENV-2 detected a particle expansion at physiological temperatures of humans, causing the E dimers to reorient with respect to each other and presenting a different surface pattern as in mosquito grown viruses ${ }^{27,28}$. bnAbs targeting the EDE will neutralize regardless of the surface arrangement of E dimers. These results are in line with a recent study on the tick-borne encephalitis flavivirus, in which the corresponding recombinant sE dimer efficiently depleted human serum from neutralizing activity ${ }^{29}$.

As a corollary, our results suggest that, similar to vaccine approaches against the respiratory syncytial virus ${ }^{30}$, a viable strategy would consist in presenting a single stabilized pre-fusion E dimer to the immune system, designed to focus the B-cell 
response on the EDE, instead of the multivalent vaccines that are currently under development. 


\section{References:}

1 Lindenbach, B., Thiel, H. \& Rice, C. Flaviviridae: the viruses and their replication. 5th edn, Vol. 1 1101-1152 ( Lippincott Williams \& Wilkins, 2007).

2 Bhatt, S. et al. The global distribution and burden of dengue. Nature 496, 504507, doi:10.1038/nature12060 (2013).

3 Capeding, M. R. et al. Clinical efficacy and safety of a novel tetravalent dengue vaccine in healthy children in Asia: a phase 3, randomised, observer-masked, placebo-controlled trial. Lancet, doi:10.1016/S0140-6736(14)61060-6 (2014).

4 Normile, D. Tropical diseases. Dengue vaccine trial poses public health quandary. Science 345, 367-368, doi:10.1126/science.345.6195.367 (2014).

5 Fibriansah, G. et al. A potent anti-dengue human antibody preferentially recognizes the conformation of $\mathrm{E}$ protein monomers assembled on the virus surface. EMBO molecular medicine 6, 358-371, doi:10.1002/emmm.201303404 (2014).

6 Teoh, E. P. et al. The structural basis for serotype-specific neutralization of dengue virus by a human antibody. Science translational medicine 4, 139ra183, doi:10.1126/scitranslmed.3003888 (2012).

7 Dejnirattisai, W. et al. A new class of highly potent broadly neutralizing antibodies isolated from dengue viremic patients. Nature Immunology in press (2014).

8 Modis, Y., Ogata, S., Clements, D. \& Harrison, S. C. A ligand-binding pocket in the dengue virus envelope glycoprotein. Proc Natl Acad Sci U S A 100, 6986-6991, doi:10.1073/pnas.0832193100 (2003).

$9 \mathrm{Yu}, \mathrm{I}$. M. et al. Structure of the immature dengue virus at low $\mathrm{pH}$ primes proteolytic maturation. Science 319, 1834-1837, doi:10.1126/science.1153264 (2008).

10 Kuhn, R. J. et al. Structure of dengue virus: implications for flavivirus organization, maturation, and fusion. Cell 108, 717-725 (2002).

11 Zhang, X. et al. Cryo-EM structure of the mature dengue virus at 3.5-A resolution. Nat Struct Mol Biol 20, 105-110, doi:10.1038/nsmb.2463 (2013).

12 Zhang, Y. et al. Conformational changes of the flavivirus E glycoprotein. Structure 12, 1607-1618, doi:10.1016/j.str.2004.06.019 (2004).

13 Cockburn, J. J. et al. Structural insights into the neutralization mechanism of a higher primate antibody against dengue virus. Embo J 31, 767-779, doi:10.1038/emboj.2011.439 (2012).

14 Rey, F. A., Heinz, F. X., Mandl, C., Kunz, C. \& Harrison, S. C. The envelope glycoprotein from tick-borne encephalitis virus at 2 A resolution. Nature 375, 291-298 (1995). 
15 Lawrence, M. C. \& Colman, P. M. Shape complementarity at protein/protein interfaces. J Mol Biol 234, 946-950, doi:10.1006/jmbi.1993.1648 (1993).

16 Cockburn, J. J. et al. Mechanism of dengue virus broad cross-neutralization by a monoclonal antibody. Structure 20, 303-314, doi:10.1016/j.str.2012.01.001 (2012).

17 Lok, S. M. et al. Binding of a neutralizing antibody to dengue virus alters the arrangement of surface glycoproteins. Nat Struct Mol Biol 15, 312-317, doi:10.1038/nsmb.1382 (2008).

18 Rodenhuis-Zybert, I. A. et al. A fusion-loop antibody enhances the infectious properties of immature flavivirus particles. J Virol 85, 11800-11808, doi:10.1128/JVI.05237-11 (2011).

19 Zhang, Y. et al. Structures of immature flavivirus particles. Embo J 22, 2604-2613, doi:10.1093/emboj/cdg270 (2003).

20 Kostyuchenko, V. A., Zhang, Q., Tan, J. L., Ng, T. S. \& Lok, S. M. Immature and mature dengue serotype 1 virus structures provide insight into the maturation process. J Virol 87, 7700-7707, doi:10.1128/JVI.00197-13 (2013).

21 Li, L. et al. The flavivirus precursor membrane-envelope protein complex: structure and maturation. Science 319, 1830-1834, doi:10.1126/science.1153263 (2008).

22 Plevka, P. et al. Maturation of flaviviruses starts from one or more icosahedrally independent nucleation centres. EMBO Rep 12, 602-606, doi:10.1038/embor.2011.75 (2011).

23 Plevka, P., Battisti, A. J., Sheng, J. \& Rossmann, M. G. Mechanism for maturationrelated reorganization of flavivirus glycoproteins. Journal of structural biology 185, 27-31, doi:10.1016/j.jsb.2013.11.001 (2014).

24 Changeux, J. P. \& Edelstein, S. Conformational selection or induced fit? 50 years of debate resolved. F1000 biology reports 3, 19, doi:10.3410/B3-19 (2011).

25 de Alwis, R. et al. Identification of human neutralizing antibodies that bind to complex epitopes on dengue virions. Proc Natl Acad Sci U S A 109, 7439-7444, doi:10.1073/pnas.1200566109 (2012).

26 Kaufmann, B. et al. Neutralization of West Nile virus by cross-linking of its surface proteins with Fab fragments of the human monoclonal antibody CR4354. Proc Natl Acad Sci U S A 107, 18950-18955, doi:10.1073/pnas.1011036107 (2010).

27 Fibriansah, G. et al. Structural changes of dengue virus when exposed to $37^{\circ} \mathrm{C}$. $J$ Virol, doi:10.1128/JVI.00757-13 (2013).

28 Zhang, X. et al. Dengue structure differs at the temperatures of its human and mosquito hosts. Proc Natl Acad Sci U S A 110, 6795-6799, doi:10.1073/pnas.1304300110 (2013). 
29 Jarmer, J. et al. Variation of the specificity of the human antibody responses after tick-borne encephalitis virus infection and vaccination. J Virol 88, 13845-13857, doi:10.1128/JVI.02086-14 (2014).

30 McLellan, J. S. et al. Structure-based design of a fusion glycoprotein vaccine for respiratory syncytial virus. Science 342, 592-598, doi:10.1126/science.1243283 (2013).

\section{FIGURE LEGENDS}

Figure 1. DENV-2 sE in complex with four EDE bnAbs.

a) Complex with Fab EDE2 A11. The sE dimer is in surface representation in side view, with the viral membrane-facing side below, colored according to domains as labeled, with foreground and background subunits in bright and pale colors, respectively. The two N-linked glycan chains at N67 and N153 are shown as ball-andstick and labeled. The A11 Fab is shown as ribbon with heavy and light chains in green and grey, respectively.

b) The unliganded DENV2 FGA02 sE dimer seen down the 2-fold axis (labeled "2"). Green and grey empty ovals (labeled VH and VL) show roughly the contact sites of heavy and light chains, respectively. Polypeptide segments relevant to the description of the epitopes are labeled.

c) "Worm" representation of the sE dimer, with the two subunits in different greys. Polypeptide segments in contact with the antibodies (either EDE1 or EDE2) are shown thicker, with the main segments of the epitope color coded as boxed in ED Fig. 4a, with additional contact segments in white or dark grey, depending on the subunit background. 
d) View down the empty arrow shown in (b), highlighting the fusion loop "valley" encased between two ridges, the $b$ strand on one subunit and the 150 loop on the other. e-g) same view as in (d) showing the complexes with bnAbs EDE2 B7 (e), EDE1 C8 (f) and EDE1 C10 (g) (only variable domains are shown). A black asterisk in (f) and (g) marks the region of the 150 loop, disordered in those complexes. Note that in the B7 and A11 complexes, the light chain is too far up to reach domain III.

\section{Figure 2. Comparison of paratopes.}

a) The paratopes (outlined in green) on the antibody surface color-coded by CDRs. The oval marks the region in contact with the E fusion loop. Note that the patch interacting with the N153 glycan (labeled) in EDE2 bnAbs corresponds to that interacting with domain III in EDE1 bnAbs.

b) Chemical nature of paratope side chains. The left column corresponds to the same orientation as in a), with the surface colored according to the side chain type. Exposed main chain atoms of heavy and light chain are in sand and white, respectively. The bottom panel shows the sE dimer in the same rendering in an "open book" orientation with respect to the antibodies. The epitope area is indicated with a black ellipse, with the fusion loop within the magenta oval corresponding to the one drawn on the antibodies.

\section{Figure 3. Exposed main chain atoms in the epitope.}

Surface representation of DENV-2 sE as viewed from outside the virion, with exposed main chain atoms orange (top panel) or with main chain atoms + conserved side chains 
in orange, and highly similar side chains in yellow (bottom panel). The epitopes of EDE1 bnAb C10 (black outline) and EDE2 bnAb B7 (green outline) are indicated, with the prM footprint outlined in blue.

\section{Captions to Extended Data Figures.}

ED Table 1. Crystallization conditions, data collection and refinement statistics.

DENV-2 sE: Den2_FGA-02 (GenBank entry KM087965). The DENV sE buffer used for all the crystallization experiments was: $150 \mathrm{mM} \mathrm{NaCl}$ and $15 \mathrm{mM}$ Tris $\mathrm{pH}$ 8. Proteins were crystallized at $18^{\circ} \mathrm{C}$. One crystal was used for each of the data sets.

*Protein concentration was estimated using theoretical extinction coefficients of the complexes (DENV sE + Fab or scFv). OD280nm of the protein solution was measured before crystallization. The theoretical extinction coefficients for individual component are: DENV-2 sE-His: 1.03; bnAb EDE2 A11 ScFv: 2.08; bnAb EDE2 B7 Fab: 1.65; bnAb EDE2 A11 Fab: 1.68; bnAb EDE1 C8 Fab: 1.52; bnAb EDE1 C10 ScFv: 2.43 (see Methods for more details). Extinction coefficients were calculated without taking into account carbohydrate moieties.

†Highest resolution shell is shown in parenthesis.

łlow-resolution for refinements was truncated to $20 \AA$.

$\S$ Ramachandran statistics were calculated with Molprobity ${ }^{31}$.

Abbreviations used: PEG MME: Poly-ethylene glycol monomethyl ether; MPD: 2-Methyl-

2,4-pentanediol; ND: non-determined; MR: molecular replacement; NCS: non- 
crystallographic symetry; TLS: parametrization describing translation, libration and screw-rotation to model anisotropic displacements.

\section{ED Table 2. Buried accessible surface areas and surface complementarity in the}

\section{various DENV sE - EDE complexes.}

BSA: Buried Surface Area $\left(\AA^{2}\right)$ of sE protein by the Fabs or ScFv (calculated with program 'areaimol' in CCP4).

SC: Shape Complementarity coefficient (calculated with program 'sc' in CCP4).

* Glycan chain contribution to BSA in $\AA^{2}$ and (\%).

† Main chain atoms contribution to BSA in $\AA^{2}$ and (\%).

‡ There are two sE dimer-(bnAb C10 ScFv $)_{2}$ complexes in the asymmetric unit.

\section{ED Fig. 1. Overall complexes and footprint of the bnAbs on the sE dimer.}

Each row corresponds to a different sE/bnAbs complex (except for the first one which shows the unliganded $\mathrm{SE}$ dimer) and each column displays the same orientation, as labeled. In the first two columns the sE dimer is depicted as ribbons and the bnAb variable domains as surface colored as in Fig.1. In the side view (left column) the viral membrane would be underneath, whereas the bottom view (middle column) corresponds to the $\mathrm{sE}$ dimer seen from the viral membrane with the antibodies visible across the sE ribbons. The top view (right column) shows the sE surface as presented to the immune system on the viral particle, showing the footprint of the antibodies (green) with a white depth-cuing fog. For clarity, a white outline delimits the green footprint on the blue surface of domain III. As a guide, in the top-left panel the glycan chains of 
foreground and background subunits are labeled in red and black respectively. The fusion loop and the ij loop are labeled on the top-middle panel, and can be seen in the other rows in contact with the bnAbs. A red star in the left panels of row c and d marks the location of the 150 loop, which is disordered in the complexes with the EDE1 bnAbs. This loop bears the N153 glycan recognized by the EDE2 bnAbs, as seen in row b, left panel (glycan shown as sticks with carbon atoms colored red). In contrast, all the bnAbs are seen contacting the N67 glycan, with C8 displaying the most contacts (row c, left panel, N67 glycan as sticks with carbon atoms yellow). A blue star in row c shows a disordered loop in domain III. Note that EDE1 C10 (row d) inserts deeper into the sE dimer than the others bnAbs.

\section{ED Fig. 2. Electrostatic potential of paratopes and the epitope on DENV-2.}

Open book representation of the complexes, with negative and positive potential displayed and colored according to the bar underneath. Because certain regions are disordered in the complexes, the unliganded DENV-2 sE dimer model, generated as described in the Online Methods section, was used to calculate the surface electrostatic potential of the sE dimer. Corresponding areas in contact are indicated by ovals as in Fig. 2.

ED Fig. 3. Unliganded bnAb A11 and EDE2 bnAbs in interactions with DENV-2 sE.

a) The structure of the unliganded EDE2 A11 scFv (red, 1.7 $\mathrm{A}$ resolution) superposed to the variable domain of Fab A11 in complex with DENV-2 sE (yellow, 3.8 Å resolution), to 
show that the same conformation is retained in the sE/Fab fragment complex.

b) Stereo view showing the superposed B7 (green) and A11 (yellow) variable domains, together with the 150 loop extracted from the structures of the corresponding Fab/DENV-2 sE complexes. Note that the main chain of the 150 loop adopts different conformations in the two complexes, mainly because of the hydroxyl group the Y99 side chain in the CDR H3 of B7 makes a hydrogen bond with sE T155. A11 has a phenylalanine at this position, and so lacks the hydroxyl group. The sE protein in the complex with A11 displays the same conformation as the unliganded sE (not shown).

c) Histograms of the atomic contacts of B7 (above the sE sequence) and A11 (below the sequence).

\section{ED Fig. 4. Residues involved in bnAb/antigen interactions.}

a) Amino acid sequence alignment of sE from the four DENV serotypes, with residues in black or light blue background highlighting identity and similarity, respectively, across serotypes. Secondary structure elements are indicated underneath, with tertiary organization given by colors as in Fig. 1 . DENV-2 sE residues contacted by the bnAbs are marked above, according to the code of the key (bottom-right insert). Full and empty symbols correspond to contacts on the reference subunit (defined as the one contributing the fusion loop to the epitope) and opposite subunit, respectively. Colored boxes highlight the 5 distinct regions of sE making up the epitopes, matching Fig. 1c. The histogram with the number of atomic contacts per sE residue by each bnAb is provided as Supplementary Information. Because the EDE2 B7 and A11 contacts are very similar, only the B7 contacts are shown here. The question mark on the 150 loop 
indicates residues likely to contact the EDE1 bnAbs, but which are not visible in the structure because the loop is disordered.

b) Sequence alignment of the four bnAbs crystallized, (in Kabat numbering ${ }^{32}$ ) and with the FRW and CDR regions in grey and white background, respectively. Blue lines over the sequence mark the CDRs in the IMGT convention ${ }^{33}$. Somatic mutations are in red with germline residues in smaller font underneath. Residues arising from the recombination process are in green. A symbol above the sequence indicates the $\mathrm{sE}$ segment contacted, according to the key of the bottom-right inset. The secondary structure elements of the EDE2 C8 Ig $\beta$-barrels are indicated above the sequence, as guide.

\section{ED Fig. 5. Epitopes and paratopes.}

The epitope area of DENV-2 sE from three different complexes is highlighted in green and dark grey, with relevant side chains as sticks, corresponding to residues interacting with the heavy and light chain, respectively (left panels). The variable domain of the corresponding bnAb is shown in side view, with interacting side chains labeled. Heavy and light chains are in dark and light grey, respectively, with somatic mutations in red and residues that arose through the recombination process (third CDR in each chain) in green. a) EDE2 B7 complex; b) EDE1 C10 complex; c) EDE1 C8 complex.

\section{ED Fig. 6. Key interactions of the bnAbs with sE}

a) The right panel shows the $\mathrm{sE}$ dimer in ribbons, with the framed area enlarged in the right panel to show the epitope, with main features labeled. b) sE dimer in complex with 
bnAb EDE2 B7, c) with EDE1 C8 and d) with EDE1 C10. The sE dimer surface is shown in a semi-transparent representation with the ribbons visible through. The glycan residues were not included in the surface, and are displayed as sticks. The relevant CDR loops of the bnAbs are shown as ribbons with side chains as sticks on top of the sE protein, colored as in Fig.1. The orientation of the left panel in rows b-d corresponds to the enlargement of row $\mathrm{a}$, and the right panel is a view along the arrow in Fig. $1 \mathrm{~b}$ (main text). Hydrogen bonds are displayed as dotted lines.

\section{ED Fig. 7. Interactions with the glycan chains.}

Ribbon representation of a) the EDE2 A11 Fab and b) the EDE1 C8 Fab in complex with DENV-2 sE, colored as in Fig. 1. The simulated annealing omit maps contoured at 1 sigma (cyan) or 0.6 sigma (gold) show clear density for the N153 (in a) and N67 glycans (in a and b) (black arrows). To create an unbiased map, all glycan atoms were removed from the structures, all B factors were reset to $20 \AA$ and the structures were re-defined using torsion dynamics simulated annealing. Note that the antibody spans the two glycans across the dimer interface (as also shown in Fig. 1). c) Views down the black arrow in a) (left panel) and the arrow in b) (right panel), through the glycan chain. The key to the sugar connectivity and nomenclature is framed at the center. d) Contacts of the sugar residues with the antibodies, coded according to the key. 
Acknowledgements: This work was made possible by a Pediatrics Dengue Vaccine Initiative (PDVI) grant to FAR, allowing to set up a production facility of recombinant DENV sE. The co-crystallization with the bnAbs was done with EU funding (DenFree consortium) to FAR and GRS/JM. FAR acknowledges support from Insitut Pasteur, from the French Government's "Investissements d'Avenir" program: Laboratoire d'Excellence 'Integrative Biology of Emerging Infectious Diseases' (grant no. ANR-10-LABX-62-IBEID, and the CNRS. GSR and JM were supported by the Medical Research Council, U.K., the Wellcome Trust, U.K., the National Institute for Health Research Biomedical Research Centre, Funding Scheme. GRS is a Wellcome Trust Senior investigator. We thank staffs at beam lines PROXIMA-1 and PROXIMA-2 at the SOLEIL synchrotron (St Aubin, France), and ID23-2 and ID29 at the European Synchrotron Radiation Facility (Grenoble, France). We thank Anavaj Sakuntabhai for coordination of the DenFree grant, Gerard Bricogne for advice on diffraction data collection strategies, Joseph Cockburn and Pierre-Yves Lozach for help with the initial sE constructs and Scott Halstead and Susy Kliks for support through the PDVI.

Author Contribution: JM, GRS and FAR conceived the experiments. WW and WD made the constructs for production of antibody fragments in S2 cells. MENS and CMK made the constructs for production of recombinant $\mathrm{sE}$, and crystallized the unliganded form of DENV-2 FGA02 sE. CGB and SP produced large amounts of sE protein for crystallization. AR and GBS prepared the recombinant bnAb fragments and $\mathrm{sE}$ for crystallization. AR and AH optimized the crystals of the complexes. PGC, WES, SD, MCV and AR collected and processed the diffraction data. PGC, MCV and SD determined the structures and refined the atomic models. PDe, FAS and FAR conceived the protocols for production of recombinant $\mathrm{SE}$. PDu provided a plasmid containing the envelope protein of DENV-2 FGA02strain circulating in French Guiana in 2002. FAR wrote the paper with the help of AR, PGC, GBS, MCV and SD.

Data Deposition: Coordinates and structure factor amplitudes have been deposited in the Protein Data Bank under accession numbers 4UTC, 4UTA, 4UT9, 4UTB and 4UT6 respectively for the structures of DENV-2 sE unliganded and in complex with EDE1 C8, EDE1 C10, EDE2 A11 and EDE2 B7, and 4UT7 for the structure of the unliganded scFv of EDE2 A11. Sequence of prM-sE fragment from Den2_FGA-02 has been deposited under GenBank entry KM087965. Patent application (UK 1413086.8) was deposited. 


\section{Methods}

\section{Recombinant SE protein production.}

Recombinant DENV-2 FGA02 sE (1-395) was cloned into a vector pMT/BIP/V5-His with a C-terminal His-tag and produced in Drosophila S2 cells ${ }^{33}$, performed essentially as described earlier for DENV-4 sE (Den4_Burma/63632/1976) ${ }^{13}$, with some modifications (see below). Briefly, sE expression was driven by the metallothionein promoter and was induced by $5 \mu \mathrm{M}$ of $\mathrm{CdCl}_{2}$ in Insect-XPRESS medium (Lonza). The constructs had a Drosophila BiP signal sequence fused at the N-terminal end of a prMsE construct for efficient translocation into the ER of the transfected S2 cells. prM was present $\mathrm{N}$-terminal to $\mathrm{sE}$, as in the $\mathrm{DENV}$ polyprotein precursor, with the $\mathrm{N}$-termini of prM and sE generated by signalase cleavage in the ER, where prM (which remains membrane-anchored) plays a chaperone role by masking the fusion loop of $\mathrm{sE}$. The $\mathrm{prM} / \mathrm{sE}$ complex is transported across the acidic compartments, where prM is cleaved by furin into pr (N-terminal half, bound to sE) and M (membrane-anchored C-terminal half). Upon reaching the external milieu, $\mathrm{sE}$ and pr dissociate, and the $\mathrm{sE}$ component is purfied by affinity chromatography from the cells' supernatant fluid. Clarified cell supernatants were concentrated 20 -fold using Vivaflow tangential filtration cassettes (Sartorius, cut-off $10 \mathrm{kDa}$ ) and adjusted to $\mathrm{pH} 8.0$, Tris $20 \mathrm{mM}$ and $500 \mathrm{mM} \mathrm{NaCl}$ before purification in an AKTA FPLC system by HisTrap-HP chromatography after buffer exchange to remove chelating agents from the culture medium. The protein was desalted after elution of the HisTrap column and further purified by ion exchange 
chromatography on MonoQ. A final purification gel filtration step using a Superdex 200 10/300 GL column equilibrated in 50mM Tris pH8, 500mM NaCl.

Note that the last 4 residues (392-395) were inadvertently replaced by a "vector" sequence "LRPL" instead of "FKKG" as in the correct DENV-2 sequence (see ED Fig. 4a). Fortunately, the "vector" residues correctly completed the $\beta$-strand G of domain III and did not introduce any detectable difference in conformation there as compared to the other available structures of DENV-2 sE. This discrepancy in the amino acid sequence has been explicitly indicated in the deposited PDB file.

\section{Production of antigen-binding ( $\mathrm{Fab}$ ) and single-chain Fv (scFv) fragments of the} bnAbs

The bnAb fragments were cloned into plasmids for expression as $\mathrm{Fab}^{34}$ and $\mathrm{scFv}^{35}$ in Drosophila S2 cells. The constructs contain a twin strep tag fused at the Cterminus (only of the heavy chain in the case of the Fab) for affinity purification. The purification protocol included streptactin affinity column followed by gel filtration as described above.

\section{Immune complex formation and isolation.}

The purified DENV-2 sE protein was mixed with Fabs or ScFvs (in $\sim 2$-fold molar excess) in standard buffer (500mM NaCl, Tris $50 \mathrm{mM}$ pH 8.0). The volume was brought to $0.2 \mathrm{ml}$ by centrifugation in a Vivaspin $10 \mathrm{kDa}$ cutoff, after $30 \mathrm{~min}$ incubation at $4^{\circ} \mathrm{C}$, the complex was separated from excess Fab or scFv by size exclusion chromatography except when a clear peak for the complex was not obtained. In this case, a molar ratio 
1:2 antigen:antibody mixture (i.e., with an excess of antibody) was directly used for crystallization. In all cases, the buffer was exchanged to $150 \mathrm{mM} \mathrm{NaCl}, 15 \mathrm{mM}$ Tris, pH 8 for crystallization trials. The protein concentrations used for crystallization, determined by measuring the optical density at $280 \mathrm{~nm}$ and using an extinction coefficient estimated from the amino acid sequences, are listed in ED Table 1.

\section{Crystallization and 3D structure determinations.}

Crystallization trials were carried out in sitting drops of 400nl. Drops were formed by mixing equal volumes of the protein and reservoir solution in the format of 96 Greiner plates, using a Mosquito robot, and monitored by a Rock-Imager. Crystals were optimized with a robotized Matrix Maker and Mosquito setups on 400nl sitting drops, or manually in 24 well plates using $2-3 \mu$ l hanging drops. The crystallization and cryo-cooling conditions for diffraction data collection are listed in ED Table 1.

X-ray diffraction data were collected at beam lines PROXIMA-1 and PROXIMA-2 at the SOLEIL synchrotron (St Aubin, France), and ID23-2 and ID29 at the European Synchrotron Radiation Facility (Grenoble, France) (ED Table 1). Diffraction data were processed using the XDS package ${ }^{36}$ and scaled with SCALA or AIMLESS ${ }^{37}$ in conjunction with other programs of the CCP4 suite ${ }^{38}$. The structures were determined by molecular replacement with PHASER $^{39}$ and / or $\mathrm{AMoRe}^{40}$ using the search models listed in ED Table 1.

Subsequently, careful model building with COOT $^{41}$, alternating with cycles of crystallographic refinement with program BUSTER/TNT ${ }^{42}$, led to a final model. 
Refinement was constrained to respect non crystallographic symmetry, and also used target restraints (with high resolution structures of parts of the complexes) and TLS refinement ${ }^{43}$ depending on the resolution of the crystal (see ED Table 1). Final omit maps were calculated using Phenix.Refine ${ }^{44}$ (ED Fig. 7).

\section{Analysis of the atomic models and Illustrations.}

Each complex was analyzed with the CCP4 suite of programs ${ }^{38}$. For intermolecular interactions, the maximal cutoff distance used for the interactions was $4.75 \AA$. Then the contacts of each residue of the Fab/ScFv or of DENV sE proteins were counted and plotted as a proportional bar above the corresponding residue.

The Ab sequences were analyzed by Abysis (www.bioinf.org.uk/software) and IMGT (www.imgt.org) ${ }^{33}$ websites for mapping CDR/FWR regions according to Kabat ${ }^{32}$ and IMGT ${ }^{33}$ conventions, respectively. The analysis of the putative germline and somatic maturation events was done with the IMGT website (www.imgt.org).

Multiple sequence alignments were calculated using ClustalW (ClustalW and ClustalX version 2 (ref. ${ }^{45}$ ) on the EBI server ${ }^{46}$. The Figures were prepared using Program ESPript ${ }^{47}$ and the PyMOL Molecular Graphics System, Version 1.5.0.4 Schrödinger, LLC. (pymol.sourceforge.net) with $\mathrm{APBS}^{48}$ and PDB2PQR tools ${ }^{49}$. For analysis purposes, we created an atomic model for the DENV-2 $\mathrm{sE}$ dimer based on chain A of sE from the complex with B7 (PDB 4UT6), which had no density breaks, in order to calculate the corresponding surface of the $\mathrm{sE}$ dimer presented in the corresponding panels in Figs 2b, 3 and ED Fig. 2. 


\section{EXTENDED REFERENCES}

31 Chen, V. B. et al. MolProbity: all-atom structure validation for macromolecular crystallography. Acta Crystallogr D Biol Crystallogr 66, 12-21, doi:10.1107/S0907444909042073 (2010).

$32 \mathrm{Wu}, \mathrm{T}$. T. \& Kabat, E. A. An analysis of the sequences of the variable regions of Bence Jones proteins and myeloma light chains and their implications for antibody complementarity. The Journal of experimental medicine 132, 211-250 (1970).

33 Lefranc, M. P. et al. IMGT, the international ImMunoGeneTics information system. Nucleic Acids Res 37, D1006-1012, doi:10.1093/nar/gkn838 (2009).

34 Backovic, M. et al. Efficient method for production of high yields of Fab fragments in Drosophila S2 cells. Protein Eng Des Sel 23, 169-174, doi:10.1093/protein/gzp088 (2010).

35 Gilmartin, A. A. et al. High-level secretion of recombinant monomeric murine and human single-chain Fv antibodies from Drosophila S2 cells. Protein Eng Des Sel 25, 59-66, doi:10.1093/protein/gzr058 (2012).

36 Kabsch, W. Xds. Acta Crystallogr D Biol Crystallogr 66, 125-132, doi:10.1107/S0907444909047337 (2010).

37 Evans, P. R. \& Murshudov, G. N. How good are my data and what is the resolution? Acta Crystallogr D Biol Crystallogr 69, 1204-1214, doi:10.1107/S0907444913000061 (2013).

38 Winn, M. D. et al. Overview of the CCP4 suite and current developments. Acta Crystallogr D Biol Crystallogr 67, 235-242, doi:10.1107/S0907444910045749 (2011).

39 McCoy, A. J. et al. Phaser crystallographic software. J Appl Crystallogr 40, 658674, doi:10.1107/S0021889807021206 (2007).

40 Navaza, J. Implementation of molecular replacement in AMoRe. Acta Crystallogr D Biol Crystallogr 57, 1367-1372 (2001).

41 Emsley, P., Lohkamp, B., Scott, W. G. \& Cowtan, K. Features and development of Coot. Acta Crystallogr D Biol Crystallogr 66, 486-501, doi:10.1107/S0907444910007493 (2010).

42 Blanc, E. et al. Refinement of severely incomplete structures with maximum likelihood in BUSTER-TNT. Acta Crystallogr D Biol Crystallogr 60, 2210-2221, doi:10.1107/S0907444904016427 (2004). 
43 Winn, M. D., Murshudov, G. N. \& Papiz, M. Z. Macromolecular TLS refinement in REFMAC at moderate resolutions. Methods in enzymology 374, 300-321, doi:10.1016/S0076-6879(03)74014-2 (2003).

44 Afonine, P. V. et al. Towards automated crystallographic structure refinement with phenix.refine. Acta Crystallogr D Biol Crystallogr 68, 352-367, doi:10.1107/S0907444912001308 (2012).

45 Larkin, M. A. et al. Clustal W and Clustal X version 2.0. Bioinformatics 23, 29472948, doi:10.1093/bioinformatics/btm404 (2007).

46 Goujon, M. et al. A new bioinformatics analysis tools framework at EMBL-EBI. Nucleic Acids Res 38, W695-699, doi:10.1093/nar/gkq313 (2010).

47 Gouet, P., Courcelle, E., Stuart, D. I. \& Metoz, F. ESPript: analysis of multiple sequence alignments in PostScript. Bioinformatics 15, 305-308 (1999).

48 Baker, N. A., Sept, D., Joseph, S., Holst, M. J. \& McCammon, J. A. Electrostatics of nanosystems: application to microtubules and the ribosome. Proc Natl Acad Sci U $S$ A 98, 10037-10041, doi:10.1073/pnas.181342398 (2001).

49 Dolinsky, T. J., Nielsen, J. E., McCammon, J. A. \& Baker, N. A. PDB2PQR: an automated pipeline for the setup of Poisson-Boltzmann electrostatics calculations. Nucleic Acids Res 32, W665-667, doi:10.1093/nar/gkh381 (2004). 
Table 1. Germline analysis of bnAbs EDE1 and EDE2.

\begin{tabular}{|c|c|c|c|c|c|c|c|c|}
\hline \multirow{2}{*}{$\begin{array}{l}\text { Heavy } \\
\text { chain }\end{array}$} & \multirow[t]{2}{*}{ V-H allele } & \multirow{2}{*}{$\begin{array}{l}\text { V-H } \\
\text { diverg" }\end{array}$} & \multirow{2}{*}{$\begin{array}{l}\text { V-H aa } \\
\text { ch/tot }\end{array}$} & \multirow[t]{2}{*}{$\mathrm{J}-\mathrm{H}$ allele } & \multirow[t]{2}{*}{ D-H allele } & \multirow{2}{*}{$\begin{array}{c}\text { CDR length } \\
{[1: 2: 3]}\end{array}$} & \multicolumn{2}{|c|}{ BSA (\%) } \\
\hline & & & & & & & CDR[1:2:3] & [CDR:FWR] \\
\hline EDE2 A11 & IGHV3-74*01 ${ }^{\dagger}$ & $8.68 \%$ & $14 / 98$ & IGHJ6*02 & IGHD3-22*01 & $\begin{array}{l}{[8: 8: 26]^{\S}} \\
{[5: 17: 24]^{\#}}\end{array}$ & $\begin{array}{l}{[6: 11: 62]^{\S}} \\
{[6: 13: 61]^{\#}}\end{array}$ & $\begin{array}{l}{[79: 3]^{\S}} \\
{[80: 3]^{\#}}\end{array}$ \\
\hline EDE2 B7 & IGHV3-74*01 & $6,94 \%$ & $9 / 98$ & IGHJ6*02 & IGHD3-22*01 & $\begin{array}{l}{[8: 8: 26]^{\S}} \\
{[5: 17: 24]^{\#}}\end{array}$ & $\begin{array}{l}{[2: 11: 63]^{\S}} \\
{[2: 14: 62]^{\#}}\end{array}$ & $\begin{array}{l}{[76: 8]^{\S}} \\
{[78: 6]^{\#}}\end{array}$ \\
\hline EDE1 C8 & IGHV3-64D*06 & $6.94 \%$ & $12 / 98$ & IGHJ6*02 & IGHD2-21*01 & $\begin{array}{l}{[8: 8: 15]^{\S}} \\
{[5: 17: 13]^{\#}}\end{array}$ & $\begin{array}{l}{[0: 14: 23]^{\S}} \\
{[0: 22: 23]^{\#}}\end{array}$ & $\begin{array}{l}{[37: 24]^{\S}} \\
{[45: 16]^{\#}}\end{array}$ \\
\hline EDE1 C10 & IGHV1-3*01 & $2.78 \%$ & $4 / 98$ & $\mathrm{IGHJ} 4^{*} 02$ & IGHD4-17*01 & $\begin{array}{l}{[8: 8: 21]^{\S}} \\
{[5: 17: 19]^{\#}}\end{array}$ & $\begin{array}{l}{[0: 9: 44]^{\S}} \\
{[0: 9: 44]^{\#}}\end{array}$ & $\begin{array}{l}{[53: 0]^{\S}} \\
{[53: 0]^{\#}}\end{array}$ \\
\hline $\begin{array}{l}\text { Light } \\
\text { chain }\end{array}$ & V-L allele & $\begin{array}{l}\text { V-L } \\
\text { diverg" }\end{array}$ & $\begin{array}{l}\text { V-L aa } \\
\text { ch/tot }\end{array}$ & J-L allele & & $\begin{array}{c}\text { CDR length } \\
{[1: 2: 3]}\end{array}$ & $\begin{array}{r}\text { BS } \\
\text { CDR[1:2:3] }\end{array}$ & $\begin{array}{l}\text { (\%) } \\
\text { [CDR:FWR] }\end{array}$ \\
\hline EDE2 A11 & IGLV2-23*01 ${ }^{\dagger}$ & $6.94 \%$ & $14 / 98$ & IGLJ3* $^{*} 02^{\dagger}$ & - & $\begin{array}{l}{[9: 3: 10]^{\S}} \\
{[14: 7: 10]^{\#}}\end{array}$ & $\begin{array}{l}{[0: 0: 4]^{\S}} \\
{[0: 10: 4]^{\#}}\end{array}$ & $\begin{array}{l}{[4: 13]^{\S}} \\
{[14: 3]^{\#}}\end{array}$ \\
\hline EDE2 B7 & IGLV2-23*01 ${ }^{\dagger}$ & $4.51 \%$ & $10 / 98$ & $\operatorname{IGLJ}^{*} 02^{\dagger}$ & - & $\begin{array}{l}{[9: 3: 10]^{\S}} \\
{[14: 7: 10]^{\#}}\end{array}$ & $\begin{array}{l}{[0: 0: 4]^{\S}} \\
{[0: 10: 4]^{\#}}\end{array}$ & $\begin{array}{l}{[4: 11]^{\S}} \\
{[14: 2]^{\#}}\end{array}$ \\
\hline EDE1 C8 & IGKV3-11*01 & $5.02 \%$ & $9 / 95$ & IGKJ2*01 & - & $\begin{array}{l}{[6: 3: 10]^{\S}} \\
{[11: 7: 10]^{\#}}\end{array}$ & $\begin{array}{l}{[10: 5: 15]^{\S}} \\
{[10: 9: 15]^{\#}}\end{array}$ & $\begin{array}{l}{[30: 9]^{\S}} \\
{[34: 5]^{\#}}\end{array}$ \\
\hline EDE1 C10 & IGLV2-14*01 & $3.82 \%$ & $10 / 98$ & IGLJ3*02 & - & $\begin{array}{l}{[9: 3: 10]^{\S}} \\
{[14: 7: 10]^{\#}}\end{array}$ & $\begin{array}{l}{[17: 8: 14]^{\S}} \\
{[17: 13: 14]^{\#}}\end{array}$ & $\begin{array}{l}{[39: 8]^{\S}} \\
{[44: 3]^{\#}}\end{array}$ \\
\hline
\end{tabular}

V-H, J-H, D-H, V-L, J-L represent the putative Homsap genes and alleles corresponding to the given bnAb, predicted by IMGT analysis (see methods).

†: Additional possibilities were also predicted by IMGT (not shown)

II: Nucleotide (nt) divergence (diverg). The total length for all V-H and V-L alleles is 288nt except for EDE1 C8 V-L (279nt)

T: Number of amino acid (aa) changes out of total V-H/V-L aa length (ch/tot).

BSA (buried surface area) for CDR and FRW regions. BSA per CDR or FRW regions are shown as percentage of the total BSA of

Fab or ScFv within the complex with DENV-2 sE. BSA are represented per individual H-CDRs [1:2:3] and L-CDRs [1:2:3] or as a

sum for light/heavy chain CDRs and FWRs [CDR:FWR].

$\S$ : IMGT and \#: Kabat definitions of CDR and FRW regions. 

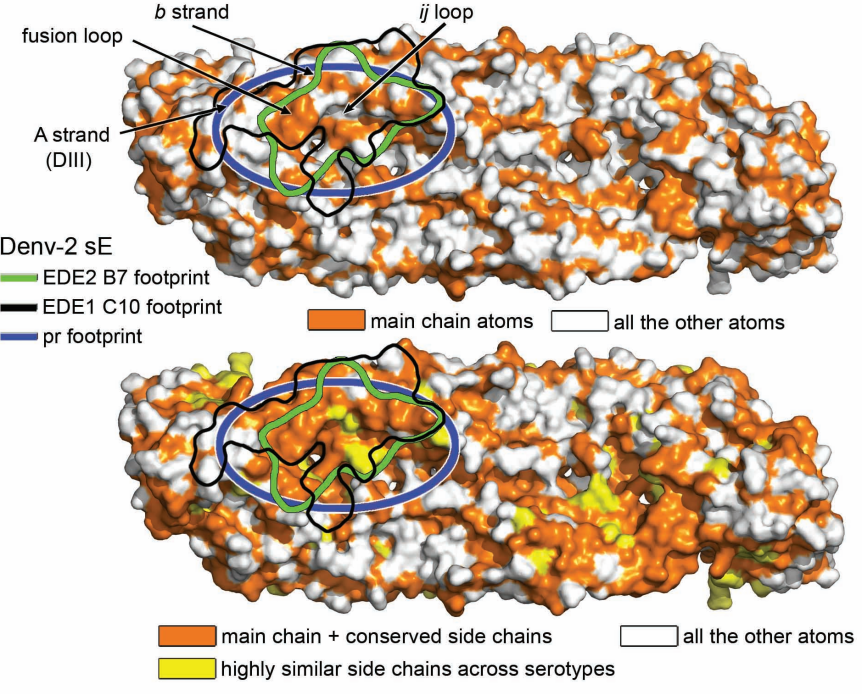


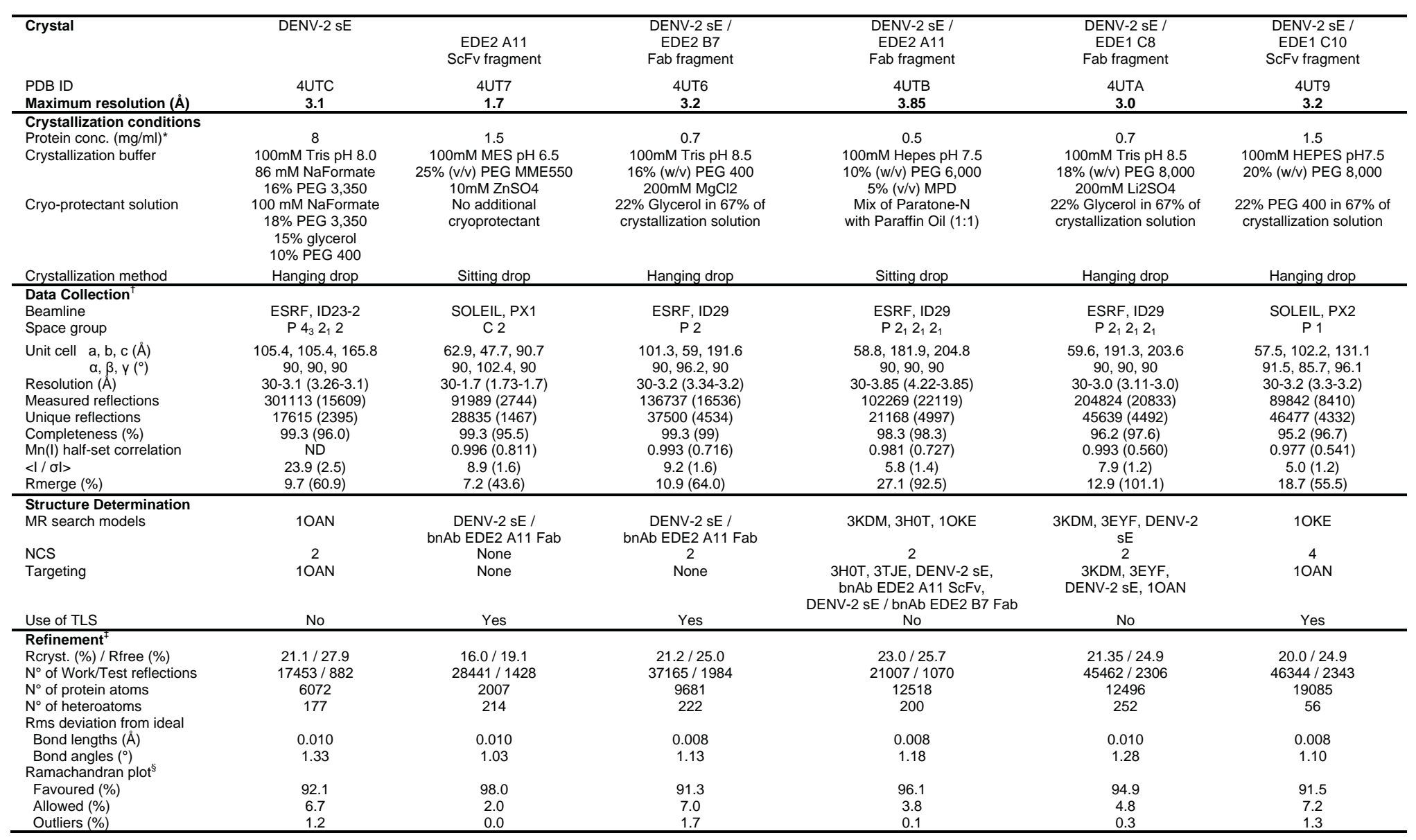




\begin{tabular}{|c|c|c|c|c|c|c|c|c|c|c|}
\hline & \multicolumn{3}{|c|}{$\begin{array}{c}\text { BSA } \\
\text { Fab or ScFv }\end{array}$} & \multicolumn{5}{|c|}{ BSA DENV-2 SE } & \multicolumn{2}{|c|}{ Complex } \\
\hline & $\mathrm{vH}$ & $\mathrm{vL}$ & Total & $\begin{array}{c}\text { Reference subunit } \\
\text { (glycans') }\end{array}$ & $\begin{array}{c}\text { Opposite subunit } \\
\text { (glycans') }\end{array}$ & Total $\left(\AA^{2}\right)$ & $\begin{array}{l}\text { Main chain } \\
\text { atoms }\left(\AA^{2}\right)^{\dagger} \\
\end{array}$ & $\begin{array}{c}\text { Total glycan } \\
\operatorname{BSA}\left(\AA^{2}\right) \\
\end{array}$ & $\begin{array}{c}\text { BSA / } \\
\text { molecule }\left(\AA^{2}\right) \\
\end{array}$ & SC \\
\hline \multicolumn{11}{|c|}{ DENV-2 sE / EDE2 B7 } \\
\hline Epitope A & 992.1 & 180.8 & 1172.9 & $621.1(83.6)$ & $478.4(358.0)$ & 1099.5 & $233(21.2 \%)$ & $442(40 \%)$ & 1136.2 & 0.728 \\
\hline Epitope B & 1010.4 & 181.5 & 1191.9 & $501.8(358.0)$ & $604.1(68.2)$ & 1105.9 & $234(21.2 \%)$ & 426 (39\%) & 1148.9 & 0.721 \\
\hline \multicolumn{11}{|c|}{ DENV-2 sE / EDE2 A11 } \\
\hline Epitope A & 945.1 & 199.4 & 1144.5 & $544.2(17.80)$ & $491.9(359.1)$ & 1036.1 & $224(21.6 \%)$ & 377 (36\%) & 1090.3 & 0.706 \\
\hline Epitope B & 984.8 & 183.2 & 1168.0 & $473.4(351.4)$ & $587.5(64.4)$ & 1060.9 & $221(20.8 \%)$ & 416 (39\%) & 1114.5 & 0.668 \\
\hline \multicolumn{11}{|c|}{ DENV-2 sE / EDE1 C8 } \\
\hline Epitope A & 744.2 & 492.3 & 1236.5 & $944.9(204.9)$ & 234.6 & 1197.6 & $362(30.2 \%)$ & $204.9(17 \%)$ & 1217.1 & 0.693 \\
\hline Epitope B & 855.7 & 559.2 & 1414.9 & 366.4 & $963.3(239.2)$ & 1329.4 & $352(26.5 \%)$ & $239.2(18 \%)$ & 1372.2 & 0.687 \\
\hline \multicolumn{11}{|c|}{ DENV-2 sE / EDE1 C10 ${ }^{\ddagger}$} \\
\hline Epitope A & 706.6 & 623.3 & 1329.9 & $781.3(84.5)$ & 366.9 & 1148.2 & $351(31 \%)$ & $84.5(7.3 \%)$ & 1239.1 & 0.681 \\
\hline Epitope B & 706.0 & 644.4 & 1350.4 & 373.2 & $778.3(94.20)$ & 1151.5 & $320(28 \%)$ & $94.2(8.2 \%)$ & 1251.0 & 0.681 \\
\hline Epitope C & 823.7 & 562.6 & 1386.3 & $717.8(90.6)$ & 465.6 & 1183.4 & $374(32 \%)$ & $90.6(7.6 \%)$ & 1284.9 & 0.742 \\
\hline Epitope D & 718.1 & 635.5 & 1353.6 & 374.9 & $788.7(99.1)$ & 1163.6 & $341(29 \%)$ & $99.1(8.5 \%)$ & 1258.6 & 0.668 \\
\hline
\end{tabular}




\section{a - DENV-2 sE}

Side view

(153) N67
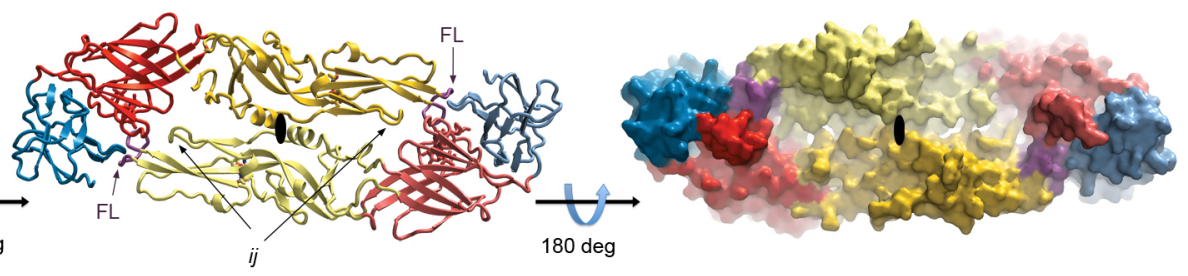

b - DENV-2 sE / EDE2 B7
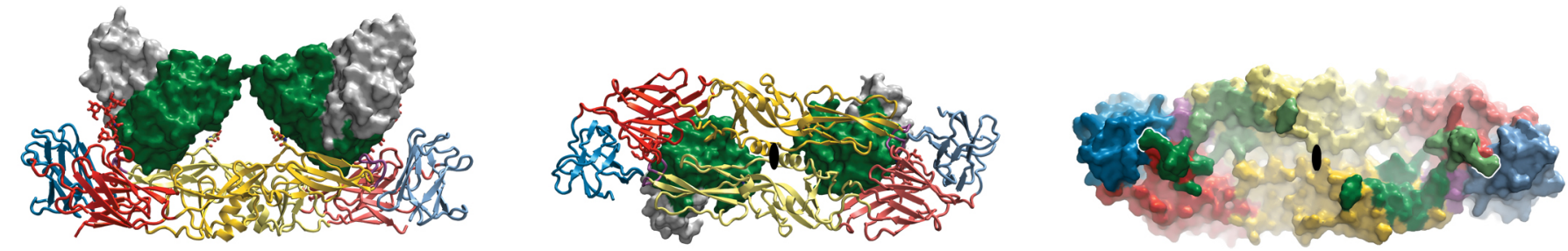

\section{c - DENV-2 SE / EDE1 C8}
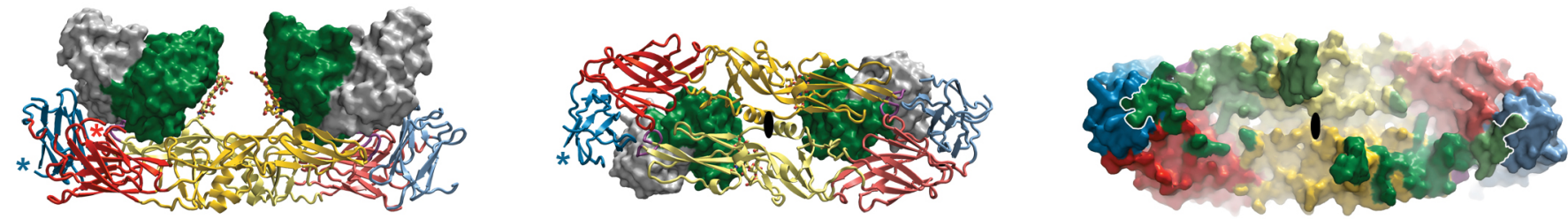

d - DENV-2 sE / EDE1 C10
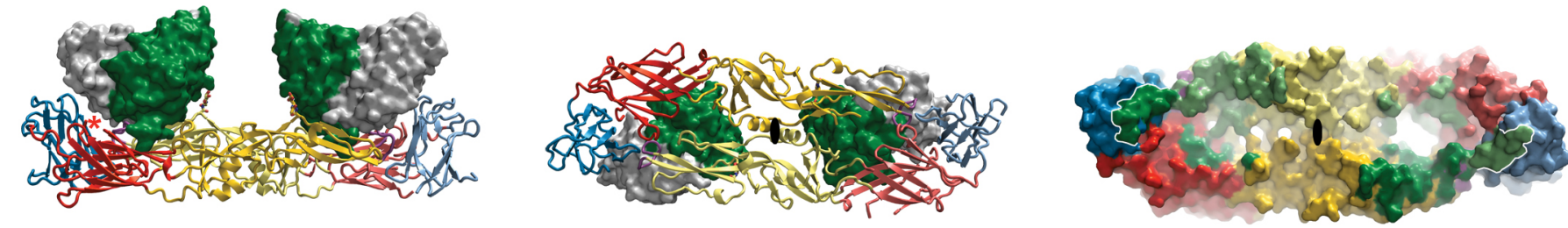
EDE2 A11

EDE2 B7

EDE1 C8
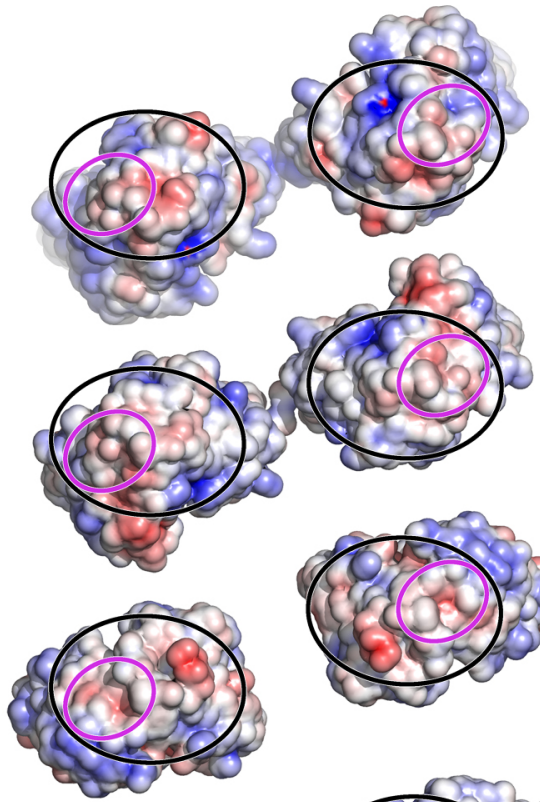

EDE1 C10
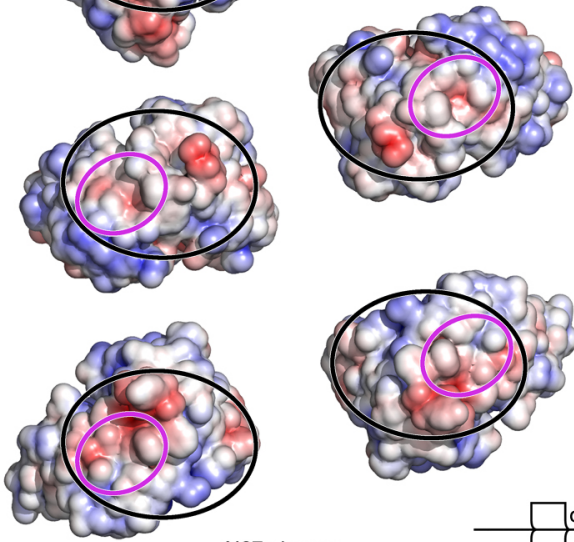

N67 glycans
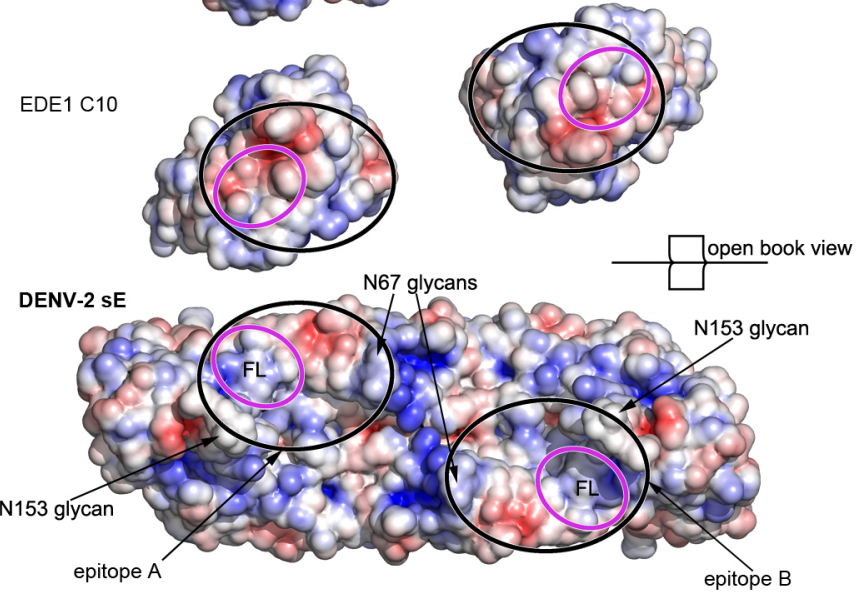
b
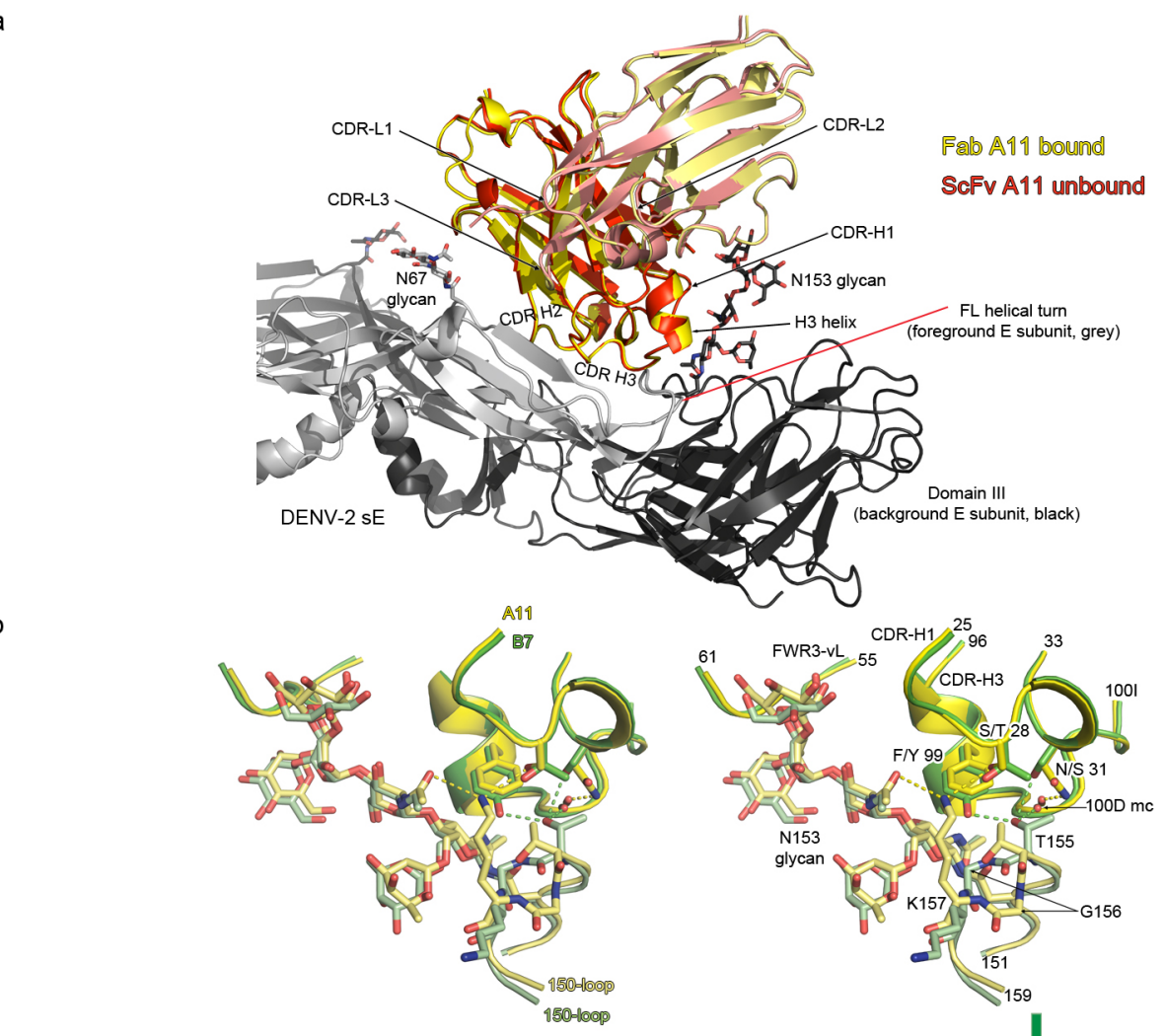

C
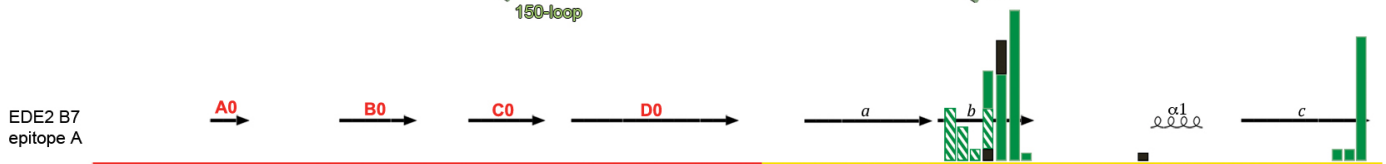

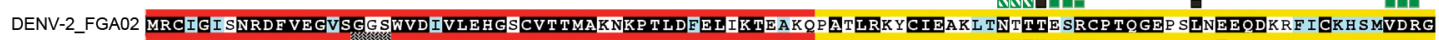
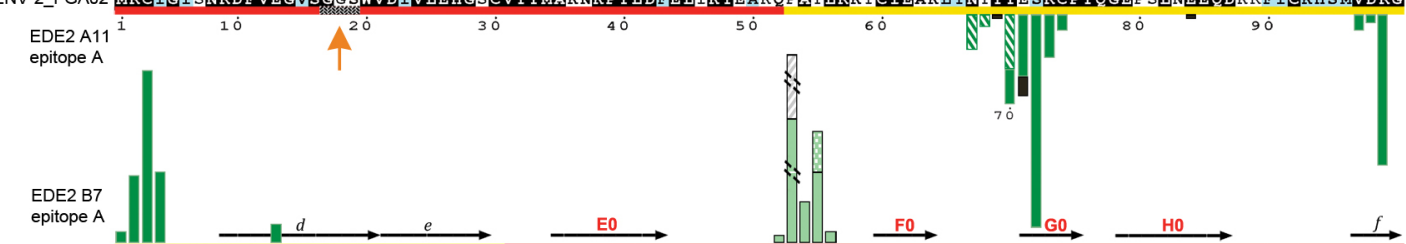

DENV-2_FGA02 WGNGCGLFGKGGIVTCAKVTCKKNMEGKIVQREENLEYTIVITPESGEEGAVGNDTGKHGKEIKITPOSSTTEAELTGYGTVTMECSPRTGLDFNEMVLLO

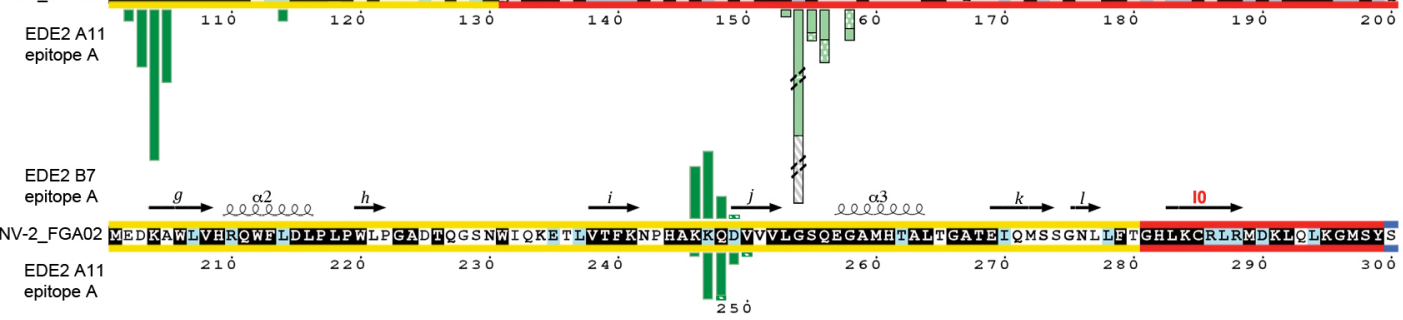

EDE2 B7

epitope $\mathrm{A} \stackrel{\mathrm{Ax}}{\rightarrow} \stackrel{\mathrm{A}}{\rightarrow} \quad \stackrel{\mathrm{B}}{\rightarrow} \quad \stackrel{\mathrm{Cx}}{\rightarrow} \stackrel{\mathrm{C}}{\rightarrow} \quad \stackrel{\mathrm{Dx}}{\rightarrow} \quad \stackrel{\mathrm{E}}{\longrightarrow} \stackrel{\mathrm{F}}{\longrightarrow} \stackrel{\mathrm{G}}{\longrightarrow}$

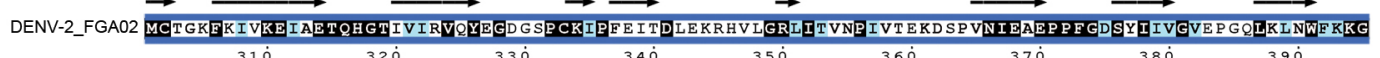

EDE2 A11

310

$330^{\circ} \quad 340^{\circ}$

$\begin{array}{llll}360^{\circ} & 370^{\circ} & 380^{\circ} & 390^{\circ}\end{array}$

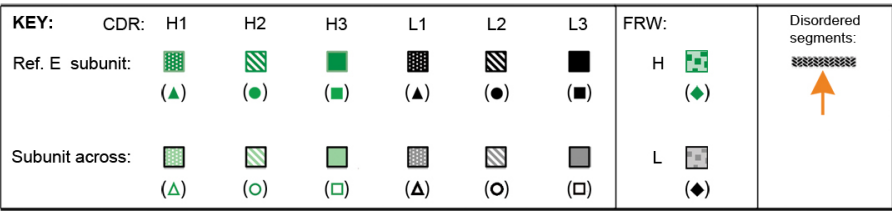


a
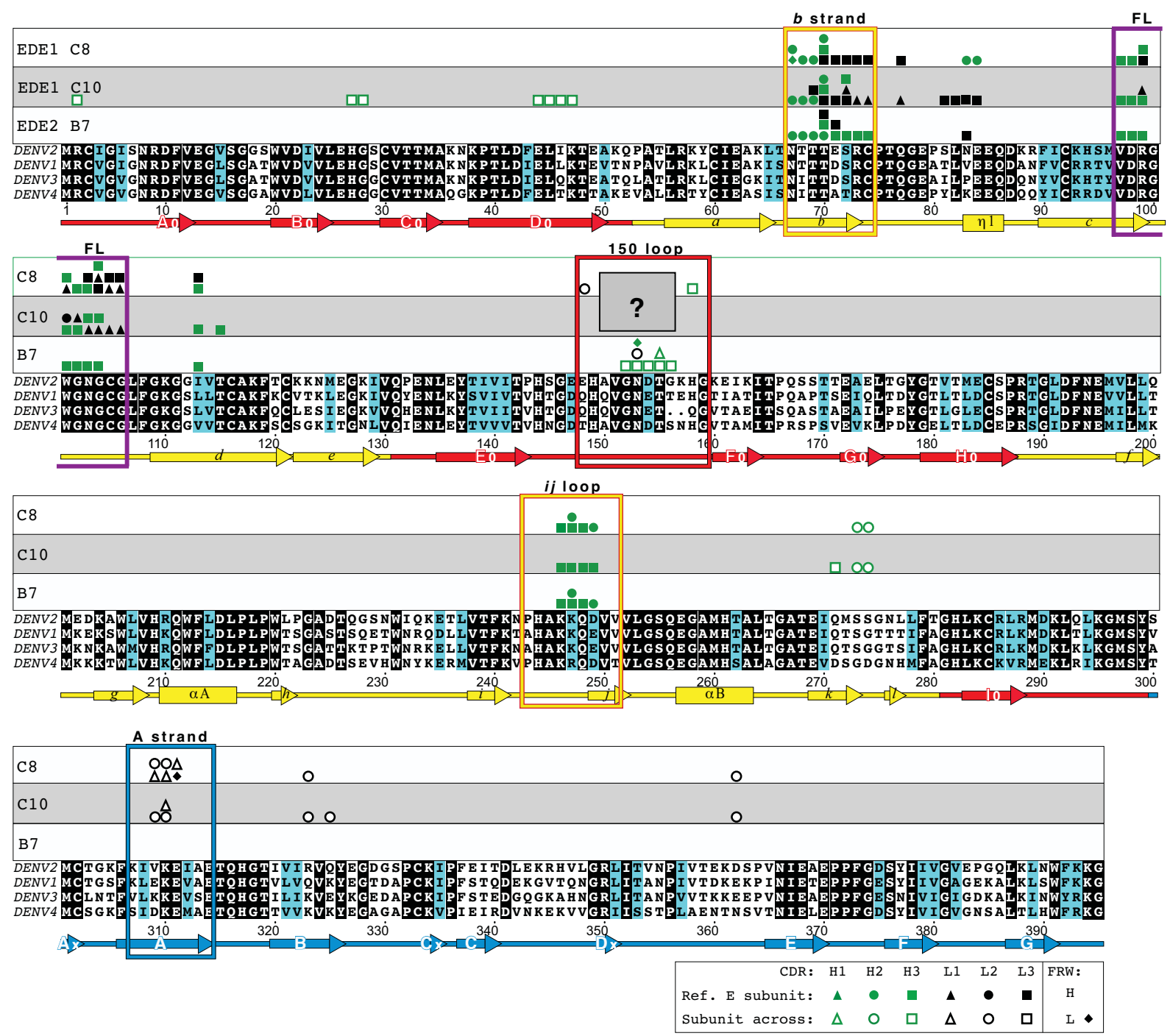

$\mathrm{b}$

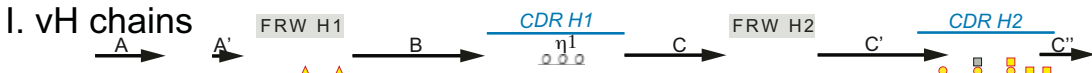

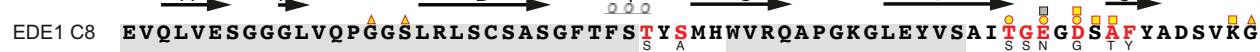
EDE1 C10 EVQLVESGAEVKKPGASVKVSCKASGYTFTSYAMHWVRQAPGQRLEWMGWINAGNGNTKY SQ QKF Q Dे

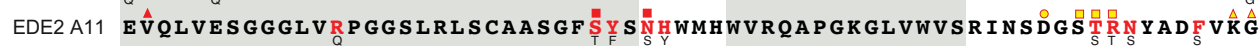

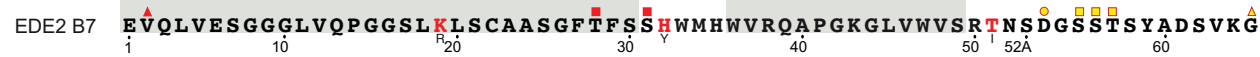

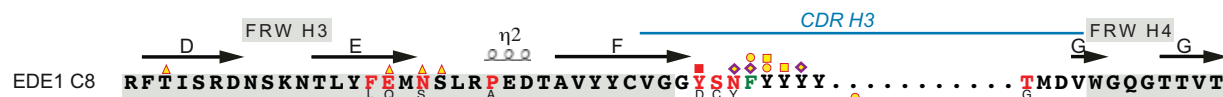
EDE1C10 RVTITRDTSASTAYMEISSIRSEDTATYYCARDKVDD Y. EDE1C10 RVTITRDTSASTAYMELSSLRSEDTATYYCARDKVDDYGDYWFF TI . . YFDYWGQGTLVTV

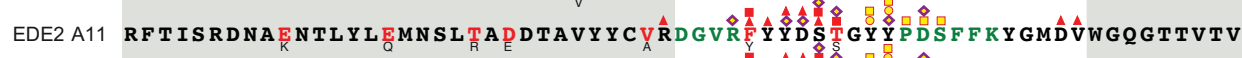

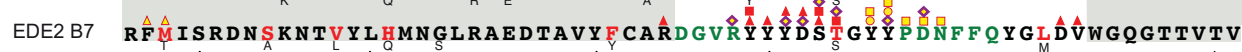
$70 \quad 80^{\circ} 82 \mathrm{ABC} \quad 90^{\circ} \quad 100^{\circ} \mathrm{ABCDEFGHIJKLMNOP}$

II. VL chains

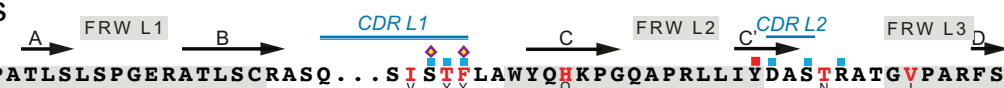

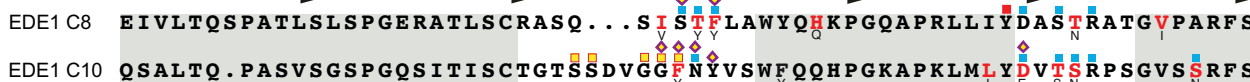

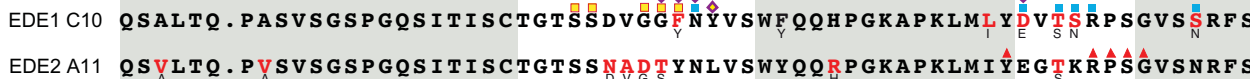

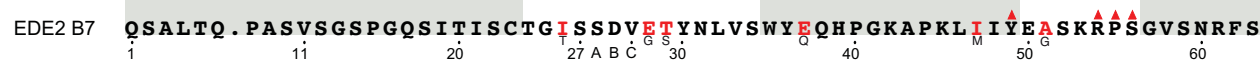

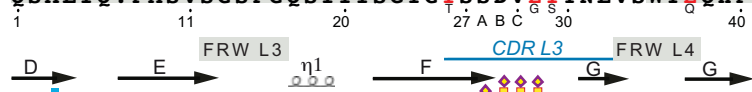
EDE1 C8 GSR R

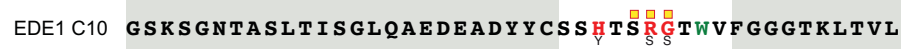

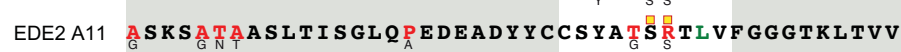
EDE2 B7 GSKSGNTASLẠI SGLQAEDEADYYCCSYAGGG ST S L FGGGTRLTVL $\square$ b-strand $\quad \triangle$ N67 glycan $\bigcirc$ ij loop $\triangle$ Fusion loop $\square \mathrm{Dl}$ across $\square \mathrm{DII}$ across DIII across

$\Delta$ N153 glycan (across) 

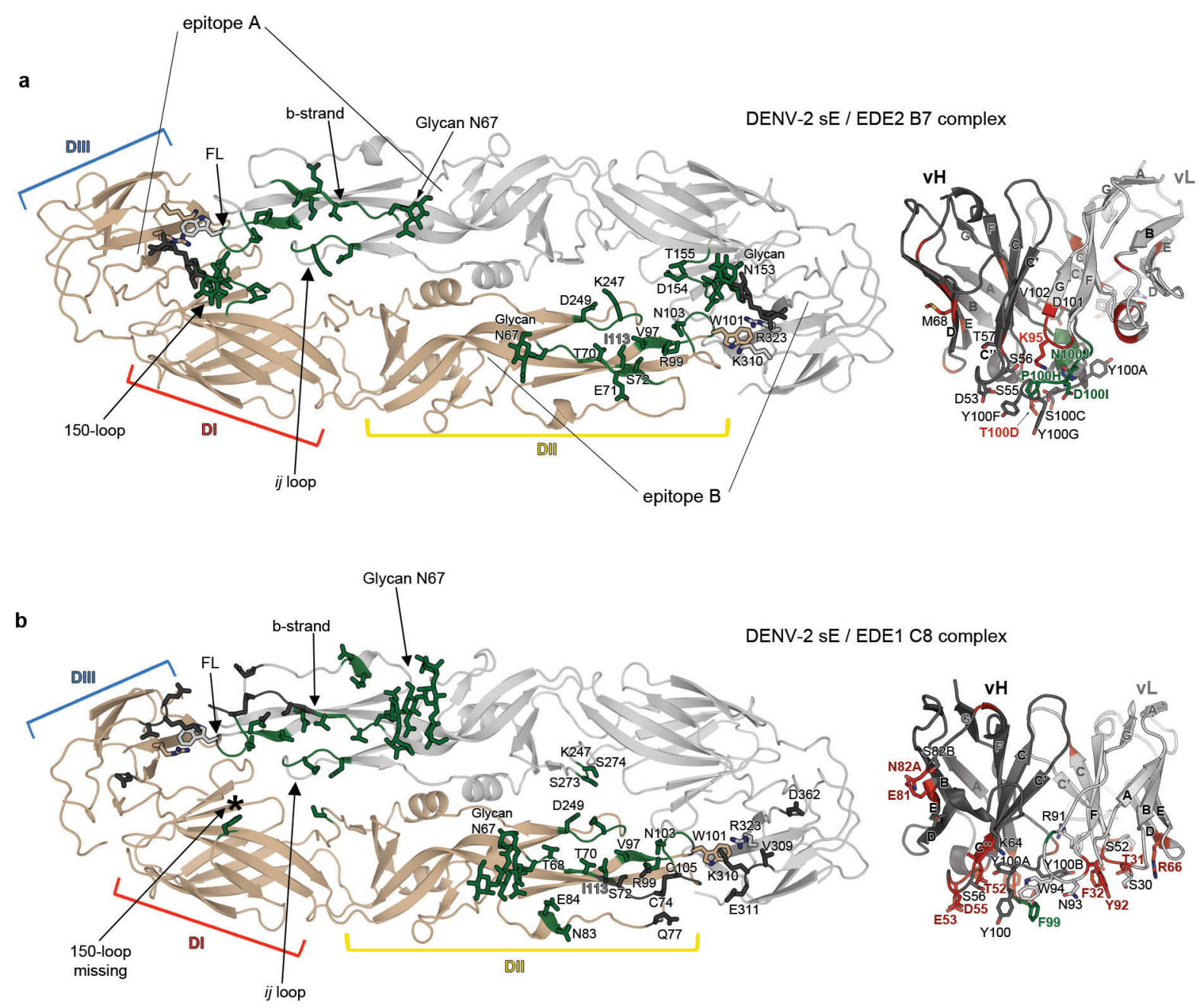

c

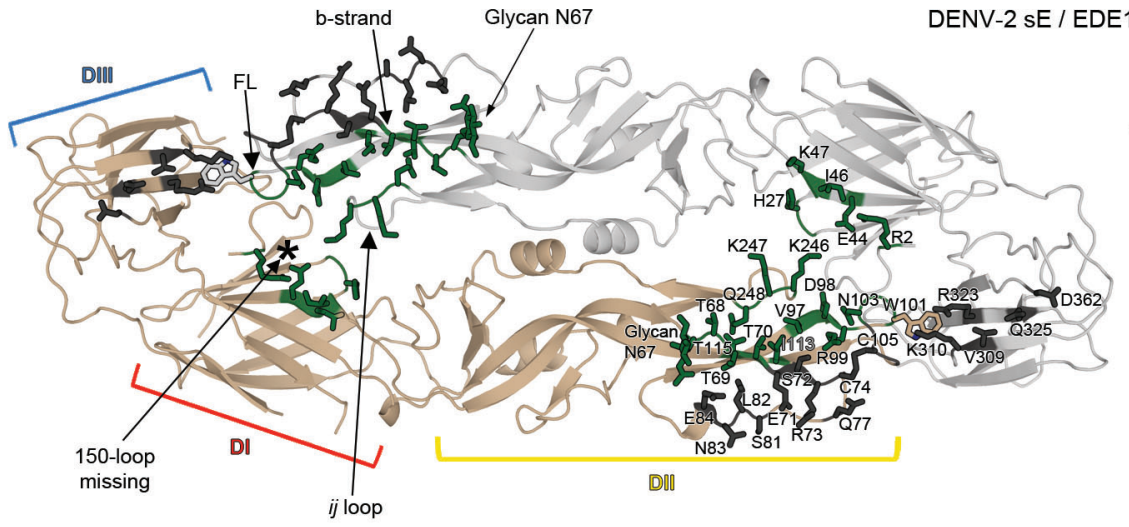

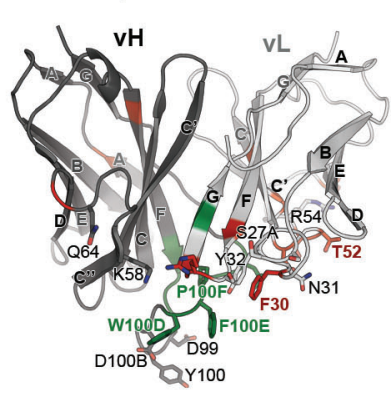




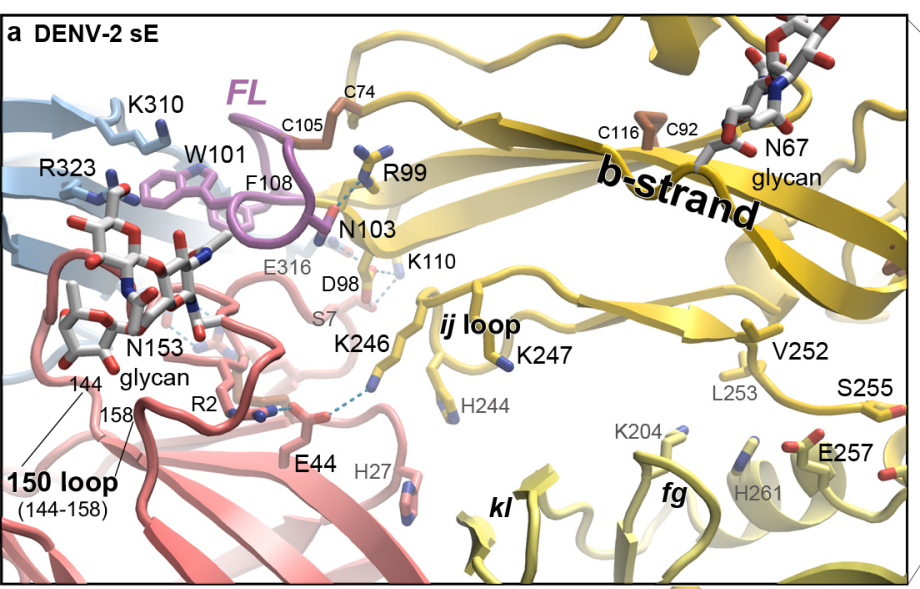

b DENV-2 SE - EDE2 B7

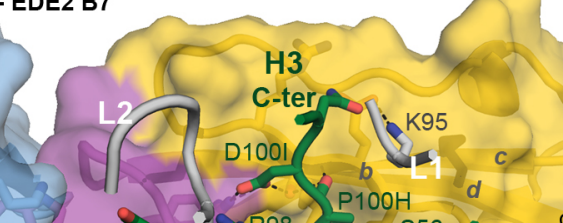
2153

glycan $30 . \mathrm{H} 3$

H1 $\mathrm{N}$-ter

\section{DENV-2 SE - EDE1 C8}

of $\mathrm{H1}$

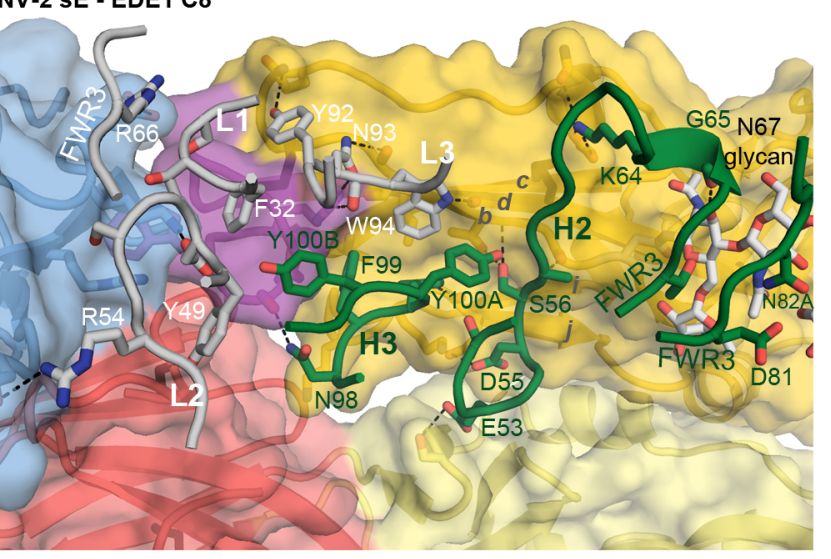

d DENV-2 SE - EDE1 C10

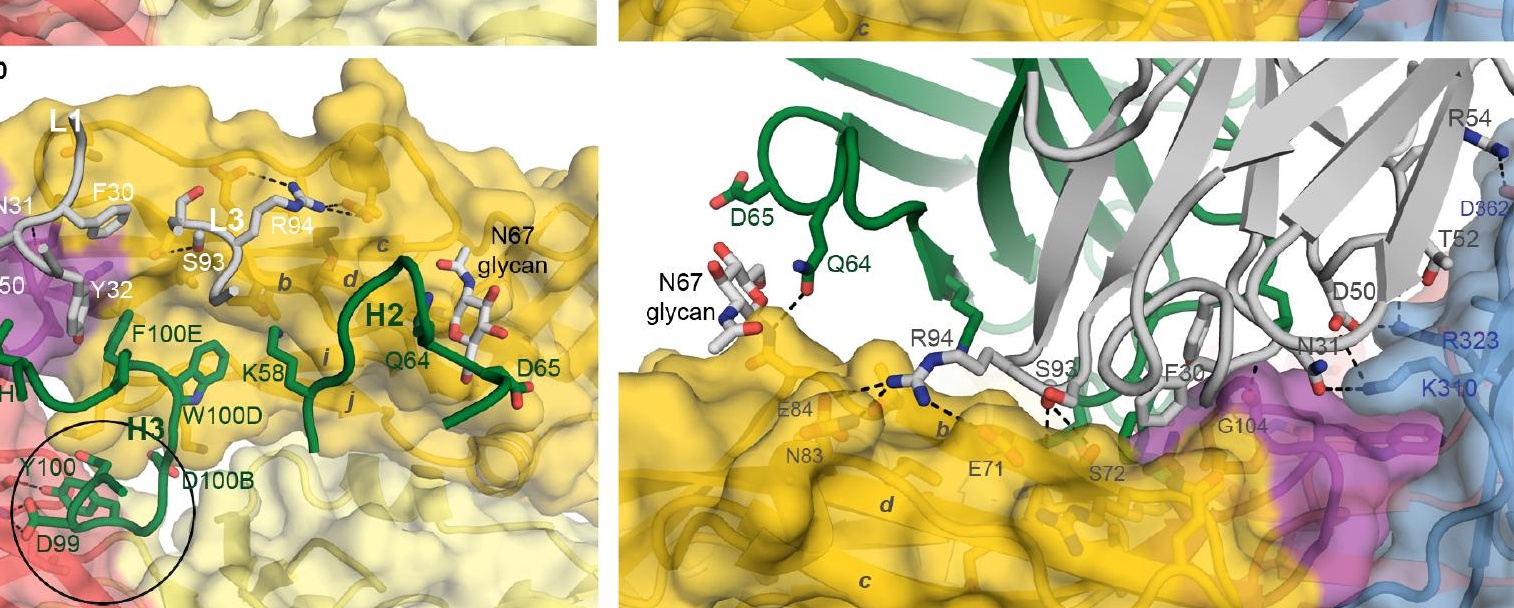

N67

glycan

DII

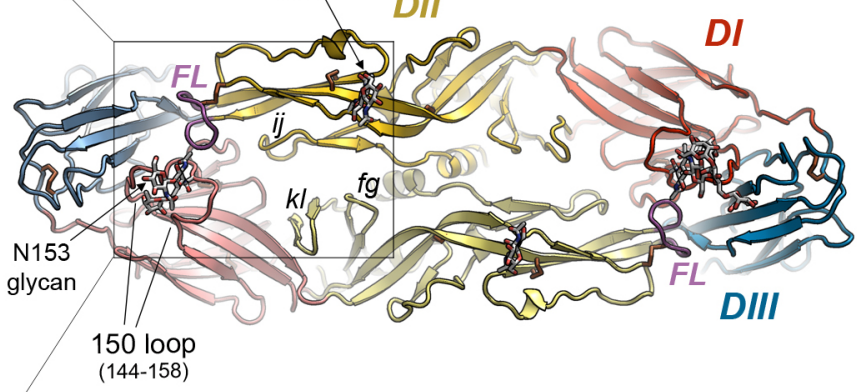

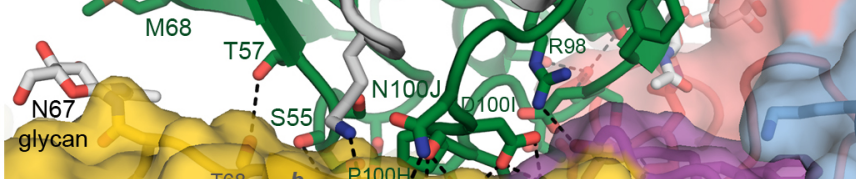
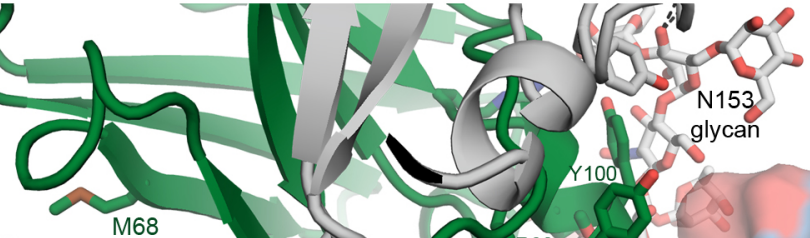

1 J ${ }_{4100}$ glycan

H FWR
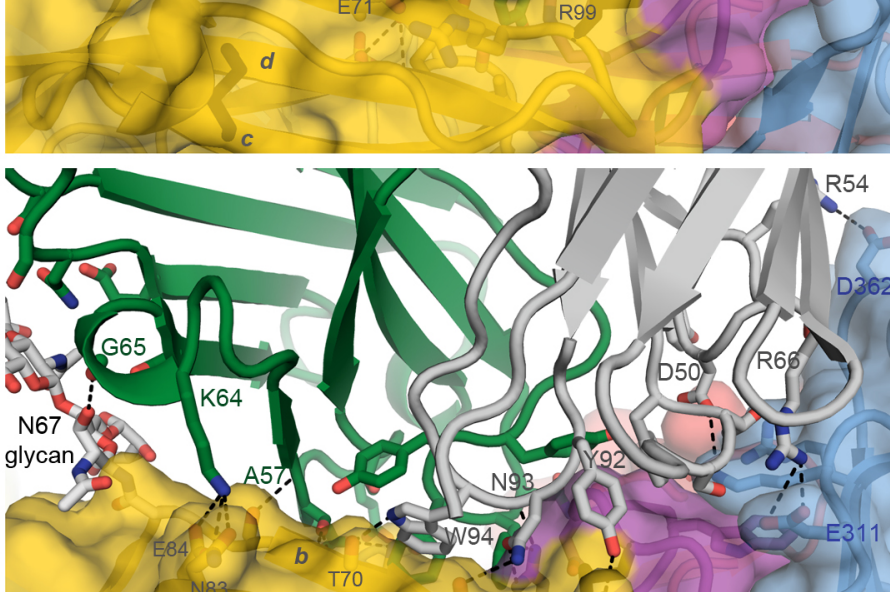


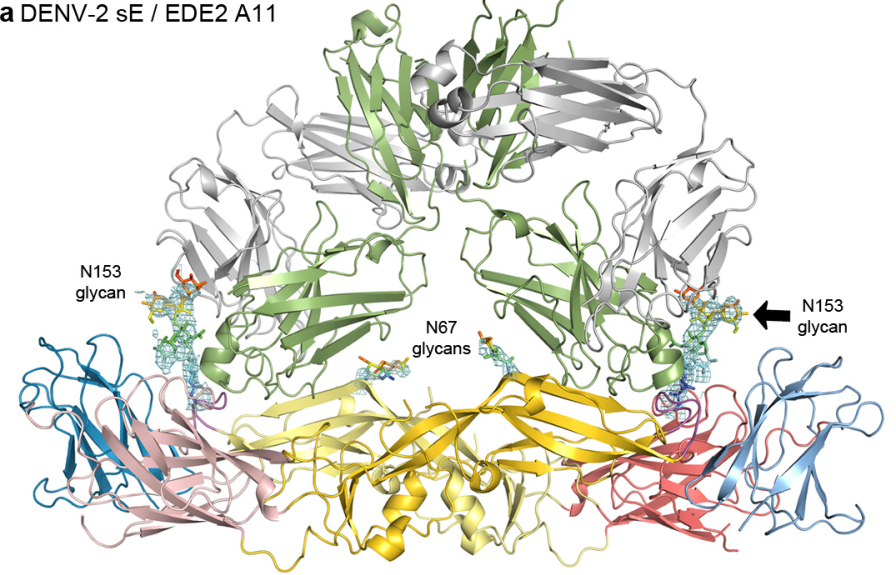

\section{b DENV-2 sE / EDE1 C8}

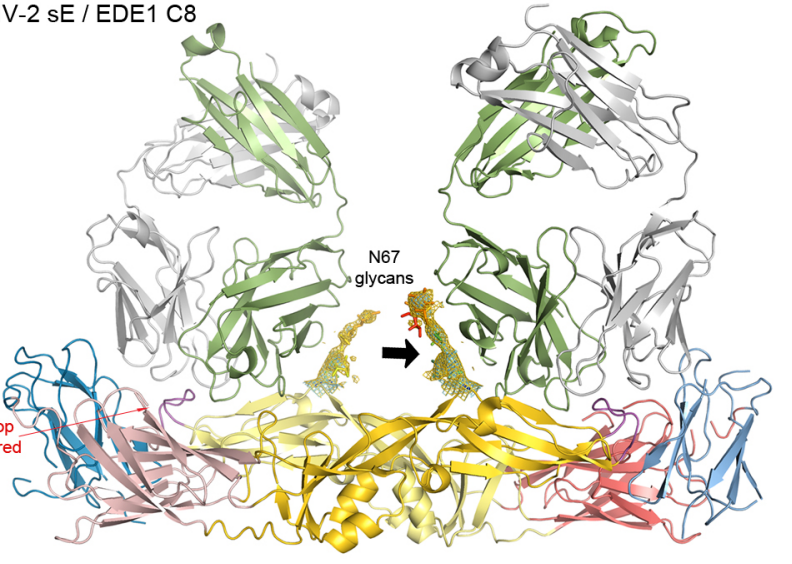

c

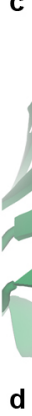
EDE1 C8
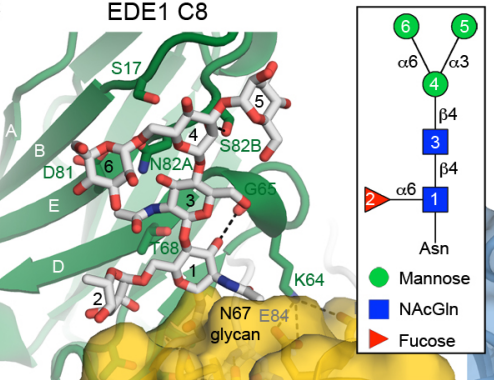

\section{Sugar contacts}

Epitope A

Epitope B

EDE1 C8

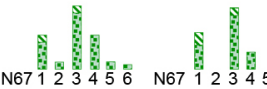
L2 $\mathbb{N}(0) \mathbb{\mathbb { N }}(0)$

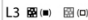
FRW:
L - 国

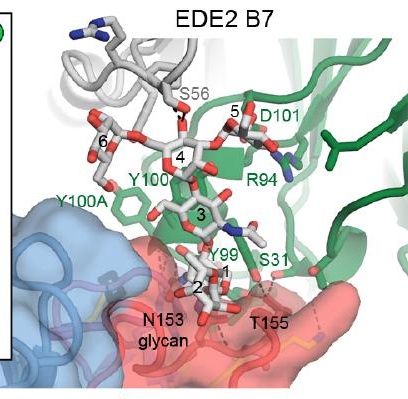

Sugar contacts

Epitope A Epitope B

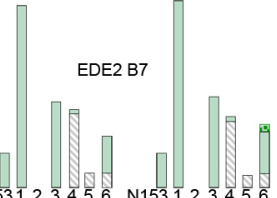




\title{
SUPPLEMENTARY INFORMATION
}

\author{
for manuscript:
}

\section{Recognition determinants of broadly neutralizing human antibodies against dengue viruses}

Alexander Rouvinski1,2*, Pablo Guardado-Calvo ${ }^{1,2^{*}}$, Giovanna Barba-Spaeth ${ }^{1,2^{*}}$, Stéphane Duquerroy ${ }^{1,2,3}$, Marie-Christine Vaney ${ }^{1,2}$, Carlos M. Kikuti ${ }^{1,2 \ddagger}$, M. Erika Navarro Sanchez ${ }^{1,2 \ddagger}$, Wanwisa Dejnirattisai ${ }^{4}$, Wiyada Wongwiwat ${ }^{4}$, Ahmed Haouz ${ }^{5}$, Christine Girard-Blanc ${ }^{5}$, Stéphane Petres ${ }^{5}$, William E. Shepard 6 , Philippe Desprès ${ }^{7 \ddagger}$, Fernando Arenzana-Seisdedos ${ }^{8}$, Philippe Dussart ${ }^{9}$, Juthathip Mongkolsapaya ${ }^{4,10 \S}$, Gavin R. Screaton ${ }^{\S}$ and Félix A. Rey ${ }^{1,2,5 \S}$

This file contains:

Page

Table of polar interactions

Histograms of antibody contacts per E protein residue:

a DENV-2 sE / EDE2 A11 complex 4

b / EDE2 B7 complex 5

c / EDE1 C8 complex 6

d / EDE1 C10 complex 7-8

Histograms of E contacts per antibody residue: 9

a DENV-2 sE / EDE2 A11 complex 10

b / EDE2 B7 complex 11

c / EDE1 C8 complex 12

d / EDE1 C10 complex 13-14 
SI Table. Polar and salt bridges interactions between DENV sE and bnAb EDE1\&2 Fab or ScFv.

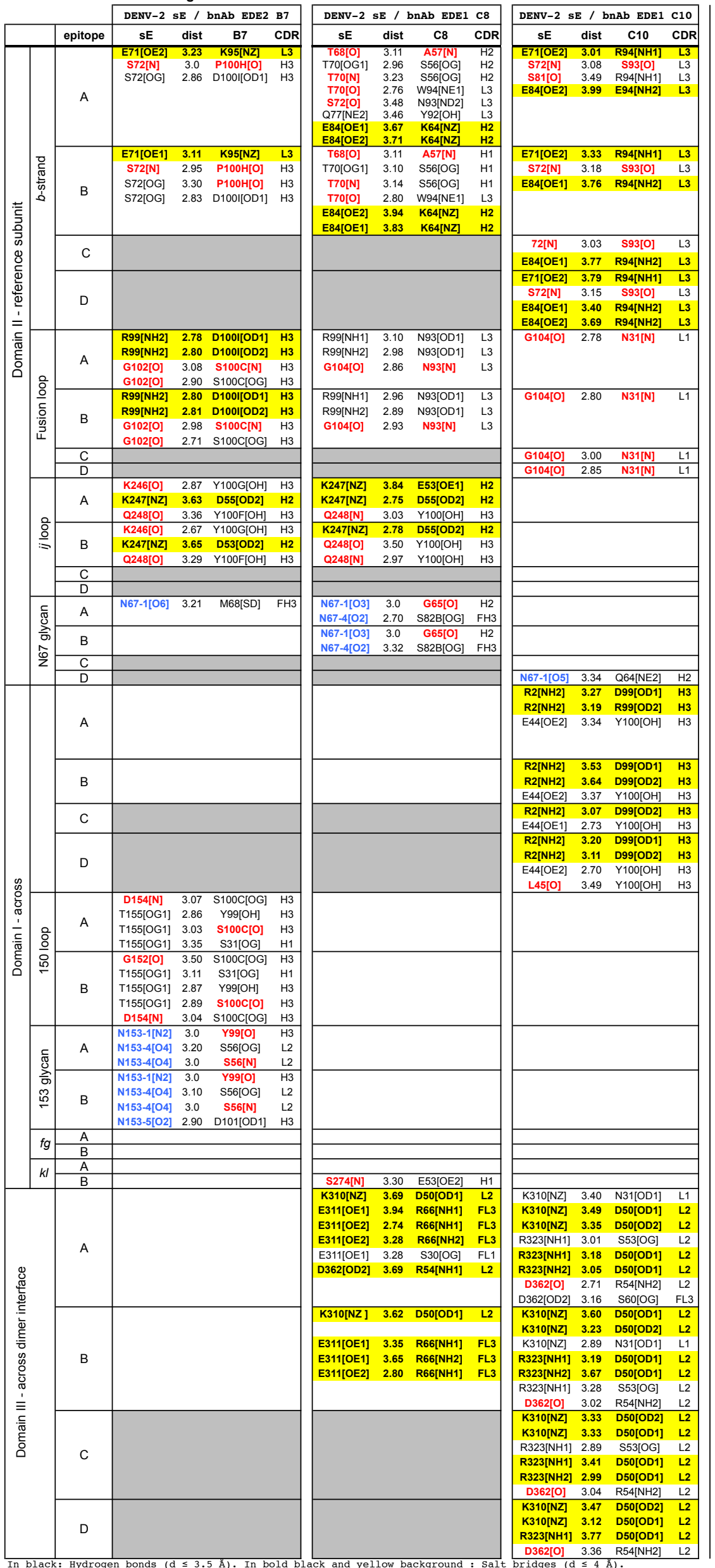

In grey background: the epitopes C and D do not exist in the corresponding stuctures. Distances(dist) are reported in $\AA$. 


\section{Histograms of antibody contacts per E protein residue:}

The following pages display the number of contacts per sE residue along its amino acid sequence, as observed in the crystal structures of all of the independent complexes in the unit cells of the crystals analyzed in the paper, as indicated.

Each page is divided in two: part (I) displays the immune complex viewed down the 2-fold axis of the sE dimer on the left (for clarity, the constant domain of the Fab fragments was removed), and on the right with the antibody removed altogether, to show the epitope. This part also defines epitopes A and B used in II. Part (II) shows the histogram of contacts corresponding to the A and B epitopes in the dimer, with the histogram bars color-coded as indicated in the key, to map the antibody region involved in the contact (in parenthesis is the symbol used in ED Fig. 4a to mark the corresponding contact). Note that the contacts pattern remains the same, but the number of contacts is not identical on the two epitopes of the sE dimer. The crystals of DENV-2 sE / EDE1 C10 had two complexes in the asymmetric unit (i.e., two sE dimers, each with two $\mathrm{C} 10 \mathrm{scFv}$ ), so that in total there are 4 independent views of the epitope, labeled A-D, described in (b). 


\section{a- DENV-2 sE / bnAb EDE2 A11 Complex}

I - 3D structure diagram with localization of epitopes
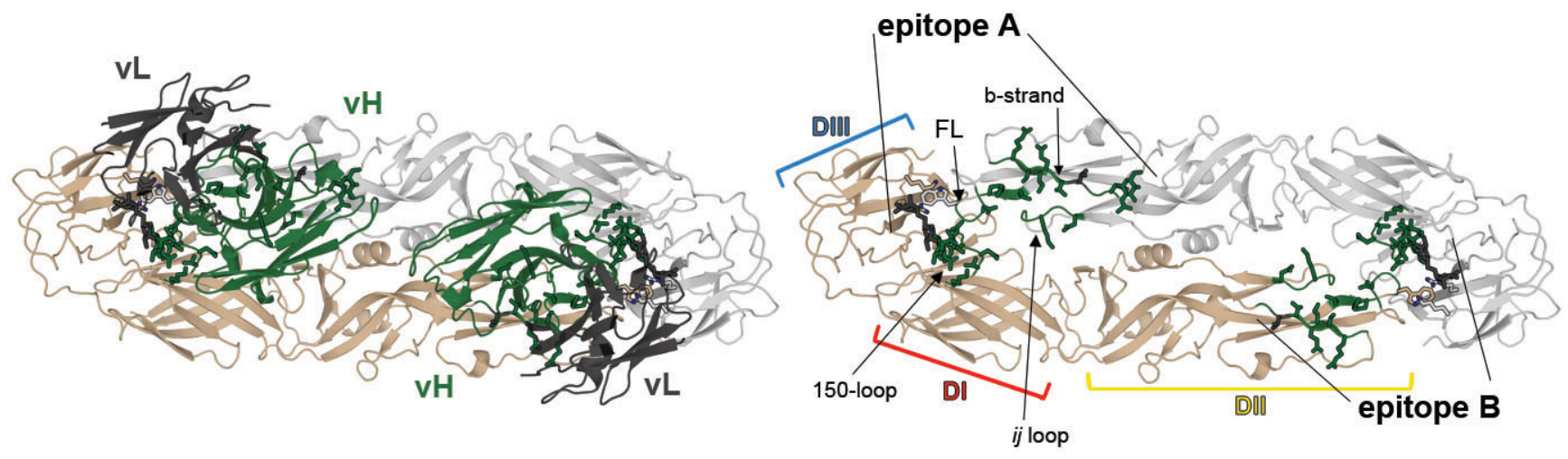

II - Histogram of number of atomic contacts of the bnAb per sE residue on epitopes $A$ and $B$

epitope A
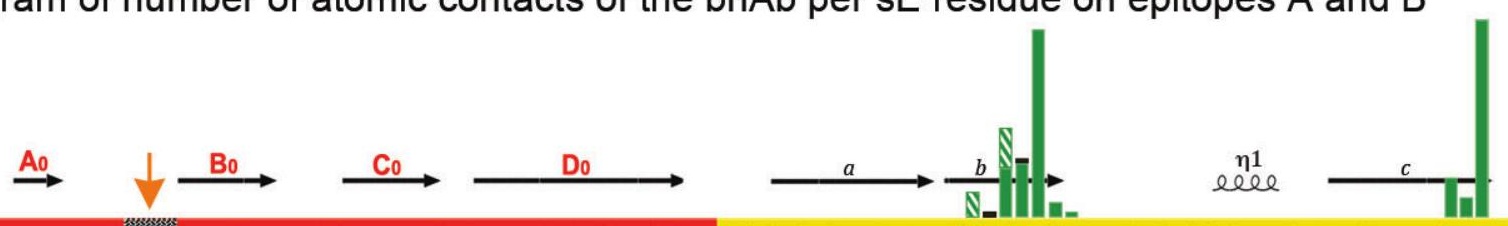

EN2_FGA02 MRCIGISNRDFVEGVSGG GSWVDIVLEHGSCVTTMAKNKP T LDFELIKTEAKQPATLRKYCIEAKL TNTTTE SRCP TQGEP SLNEEQDKRF ICKH S MVDRG

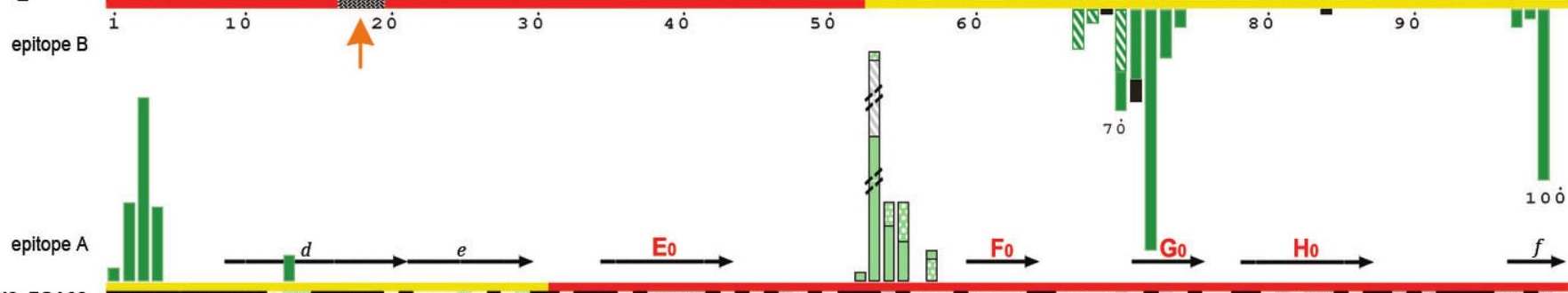

DEN2_FGA02 WGNGCGLFGKGGIVTCAKFTCKKNMEGK IVQPENLEYTIVITPHSGEEHAVGNDTGKHGKE I KI TPQSSTTEAELTGYGTVTMECSPRTGLDFNEMVLLQ

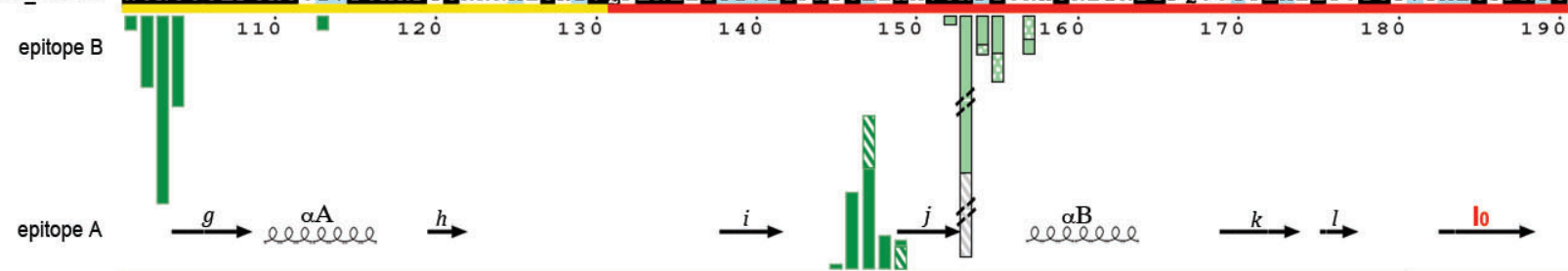

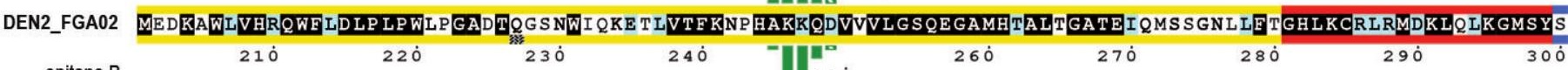

epitope B

$210^{\circ} 220^{\circ} \quad 230^{2} \quad 240^{\circ}$

260

epitope $\mathrm{A} \stackrel{\mathrm{Ax}}{\rightarrow} \stackrel{\mathrm{A}}{\rightarrow} \stackrel{\mathrm{B}}{\rightarrow} \quad \stackrel{\mathrm{Cx}}{\rightarrow} \stackrel{\mathrm{C}}{\rightarrow} \quad \stackrel{\mathrm{Dx}}{\rightarrow} \stackrel{\mathrm{E}}{\longrightarrow} \stackrel{\mathrm{F}}{\longrightarrow} \stackrel{\mathrm{C}}{\longrightarrow}$ DEN2_FGA02 MCTGKEKIVKEIAETQHGTIVIRVQYEGD GSPCKIPFE ITDLEKRHVLGRLITVNPIVTEKD SPVNIEAEP PFGDSYIIVGVEPGQLKLNWFKKG

epitope B

$\begin{array}{llllllll}310^{\circ} & 320^{\circ} & 330^{\circ} & 340^{\circ} & 350^{\circ} & 360^{\circ} & 370^{\circ} & 380^{\circ}\end{array}$

\begin{tabular}{|c|c|c|c|c|c|c|c|}
\hline KEY: CDR: H1 & $\mathrm{H} 2$ & H3 & L1 & L2 & L3 & FRW: & $\begin{array}{l}\text { Disordered } \\
\text { segments: }\end{array}$ \\
\hline 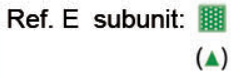 & (e) & (回) & 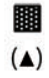 & $\begin{array}{l}\mathbb{N} \\
\text { (•) }\end{array}$ & $(\square)$ & 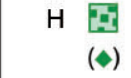 & sazasas: \\
\hline Subunit across: & $\underset{\text { (o) }}{\mathbb{Q}}$ & $\begin{array}{l}\square \\
(\square)\end{array}$ & $\begin{array}{l}\text { 罒 } \\
(\boldsymbol{\Delta})\end{array}$ & $\begin{array}{l}\mathbb{N} \\
(0)\end{array}$ & $\begin{array}{l}\square \\
(\square)\end{array}$ & L & \\
\hline
\end{tabular}




\section{b- DENV-2 sE / bnAb EDE2 B7 Complex}

I - 3D structure diagram with localization of epitopes
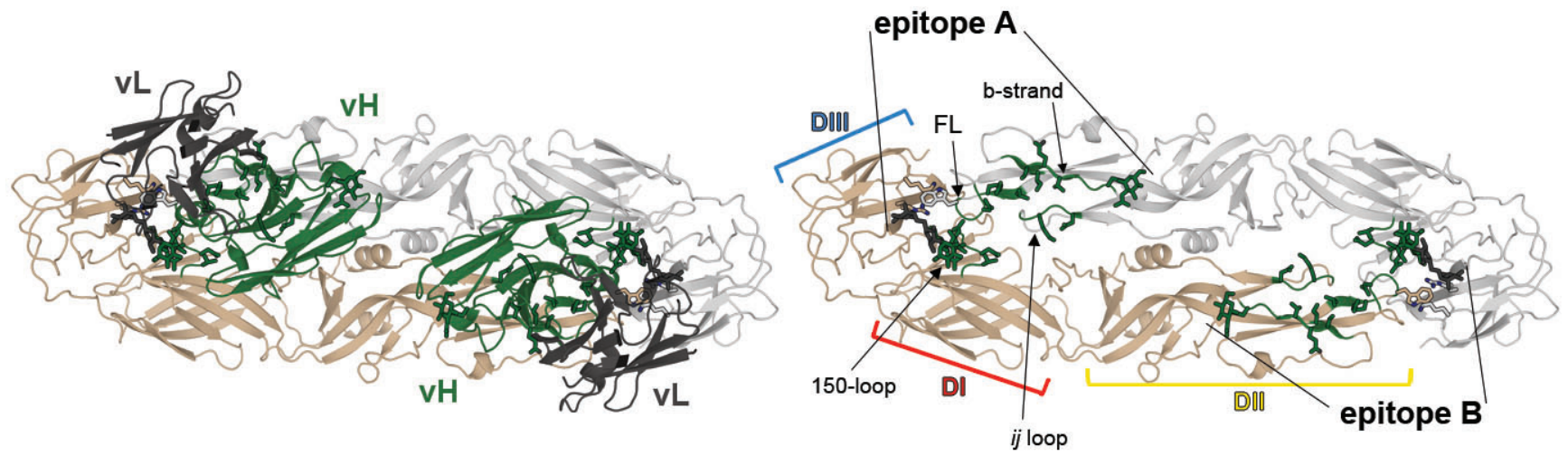

II - Histogram of number of atomic contacts of the bnAb per sE residue on epitopes A and B

epitope A
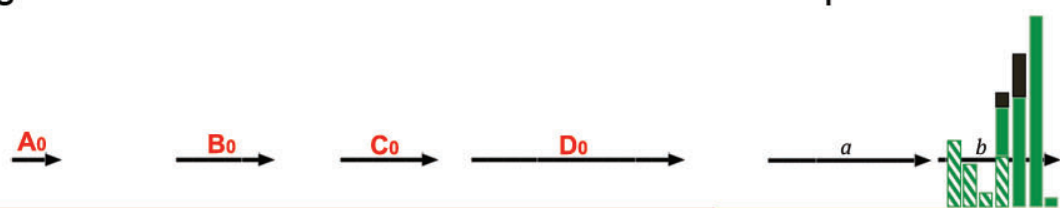

DEN2_FGA02

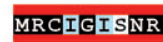

epitope B
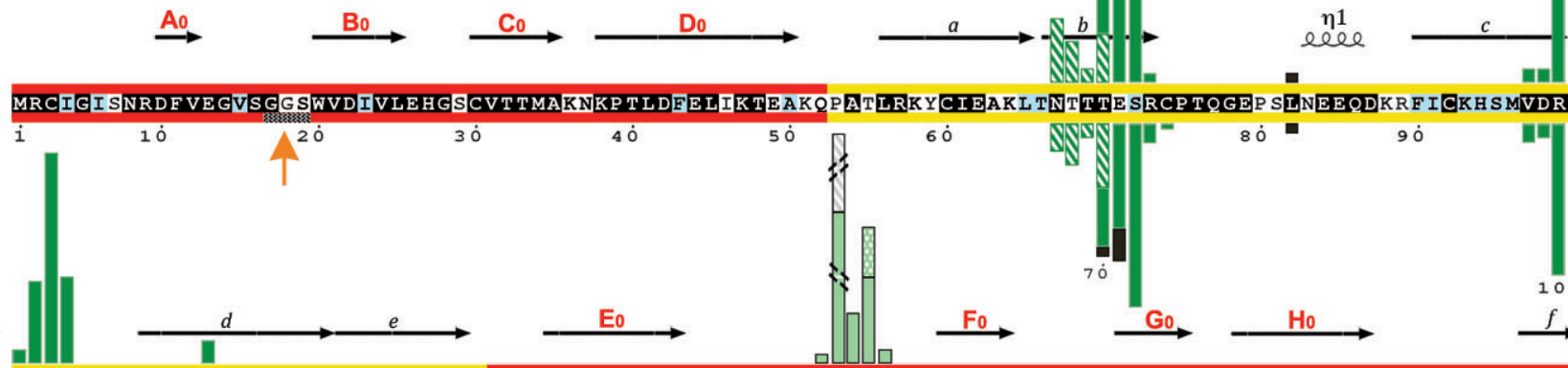

epitope A
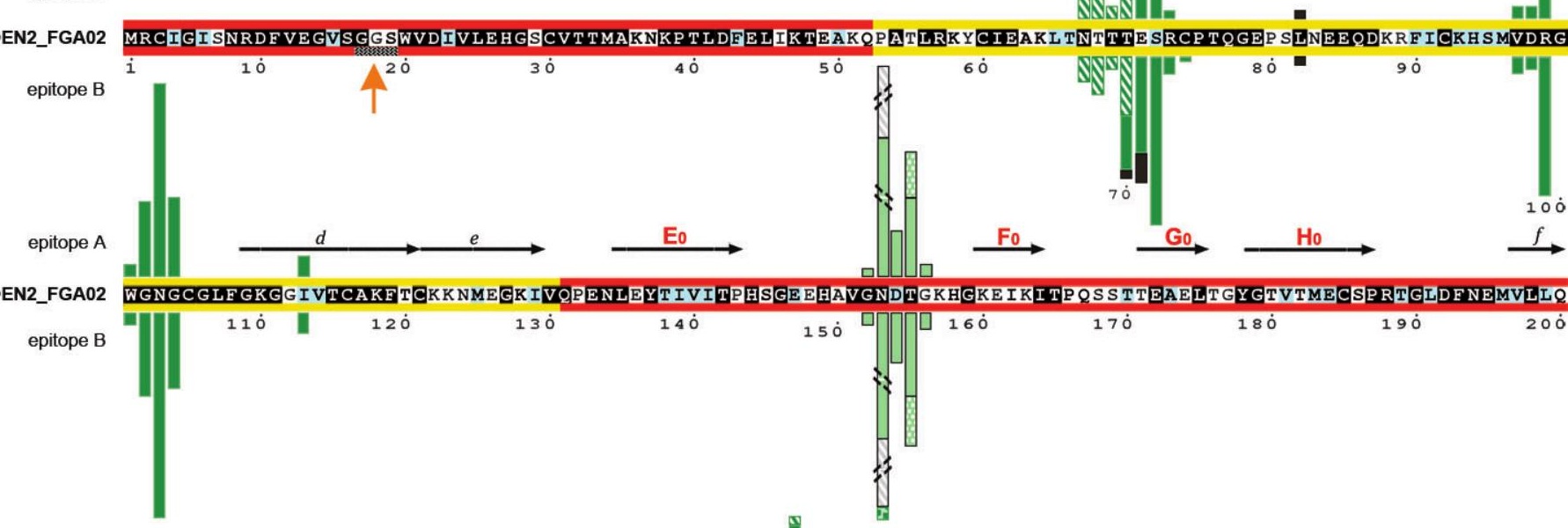

epitope A
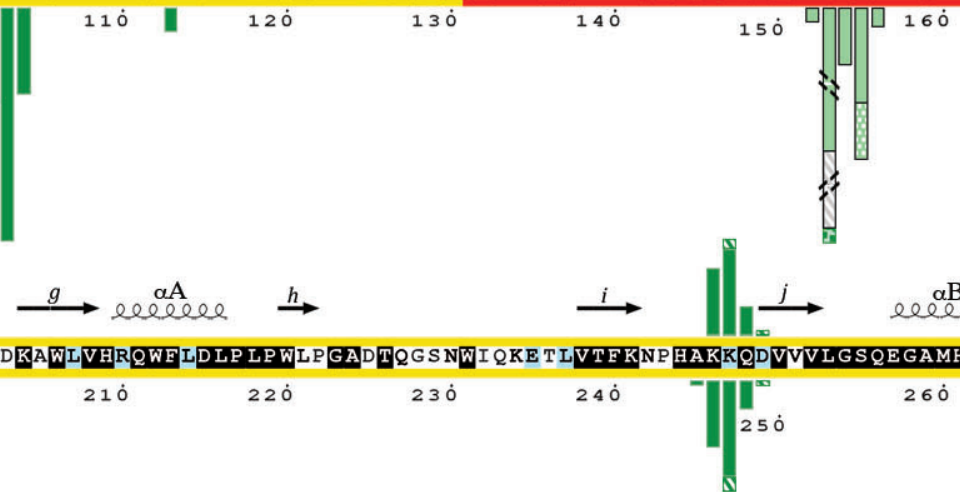

DEI

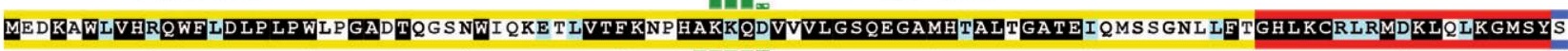

epitope B

210

220

230

$260 \dot{0}$

270 '

$280^{\circ}$

$290^{\circ}$

300

epitopen

DEN2_FGA02

epitope B

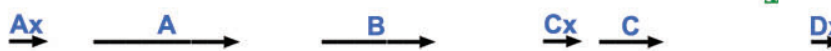

Dx

E

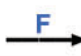

$\stackrel{\mathrm{G}}{\longrightarrow}$

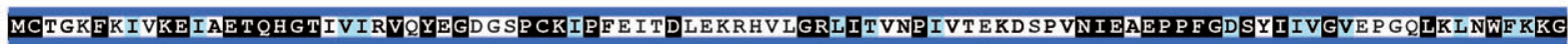

$320^{\circ}$

$330^{\circ}$

340

$350^{\circ}$

$360^{\circ}$

$370^{\circ}$

$380^{\circ}$

$390^{\circ}$

\begin{tabular}{|c|c|c|c|c|c|c|c|}
\hline $\begin{array}{l}\text { KEY: CDR: H1 } \\
\text { Ref. E subunit: 聞 } \\
\end{array}$ & $\begin{array}{l}\mathrm{H} 2 \\
\mathbb{N} \\
\text { (•) }\end{array}$ & $\begin{array}{l}\text { H3 } \\
\text { (অ) }\end{array}$ & $\begin{array}{l}\text { 聞 } \\
(\boldsymbol{\Lambda})\end{array}$ & $\begin{array}{l}\text { L2 } \\
\mathbb{N} \\
(\bullet)\end{array}$ & (घ) & $\begin{aligned} \text { FRW: } & \\
\mathrm{H} & (\diamond)\end{aligned}$ & 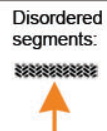 \\
\hline Subunit across: & $\underset{(0)}{\mathbb{Q}}$ & $\begin{array}{l}\square \\
(\square)\end{array}$ & $\begin{array}{l}\text { 国 } \\
(\Delta)\end{array}$ & $\begin{array}{l}\mathbb{Q} \\
(\mathbf{0})\end{array}$ & $\begin{array}{l}\square \\
(\square)\end{array}$ & L $\frac{\square}{(\bullet)}$ & \\
\hline
\end{tabular}




\section{d - DENV-2 sE / bnAb EDE1 C10 Complex}

I - 3D structure diagram with localization of epitopes

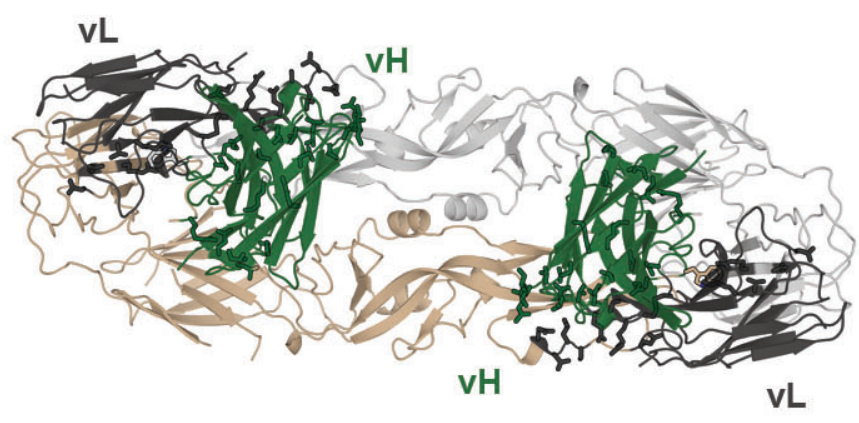

vL

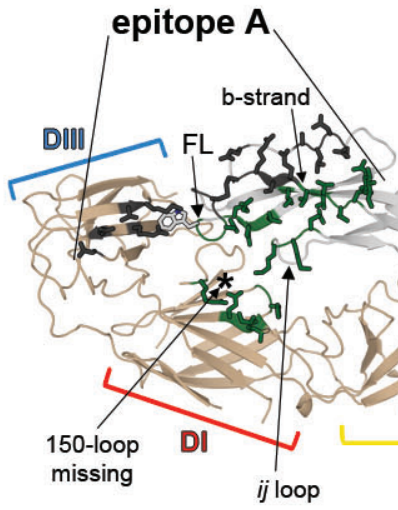

ijloop $\ln 2$

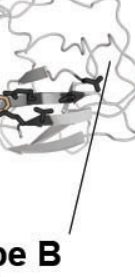

II - Histogram of number of atomic contacts of the bnAb per sE residue on epitopes $A$ and $B$

epitope A

DEN2_FGA02

EN2_FGA02

epitope B

DEN2_FGA02

epitope B

epitope A

EN2_FGA02

epitope B
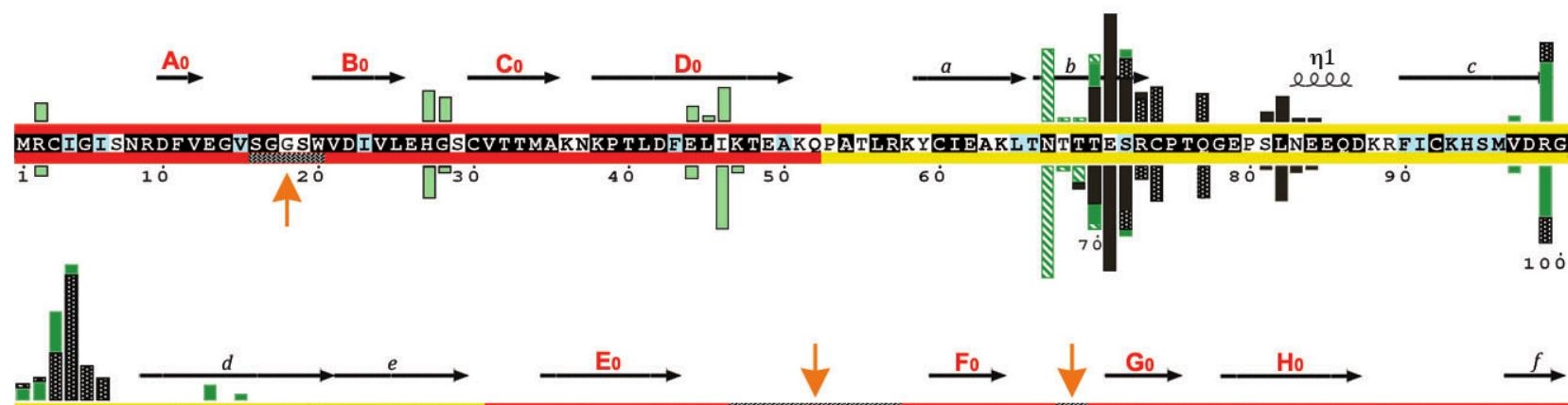

$\downarrow$

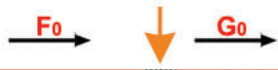

$\mathrm{Ho}$

WGNGCGLFGKG G IVTCAKETCKKNMEGK IVQPENLEYTIVITPGSGE E HAV GNDT GKHGKE IKITPQSS TTEAELTGYGTVTMECSPRTGIDFNEMVLLO

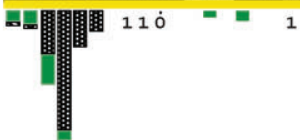

150

160

170

$180^{\circ}$

$190^{\circ}$

200

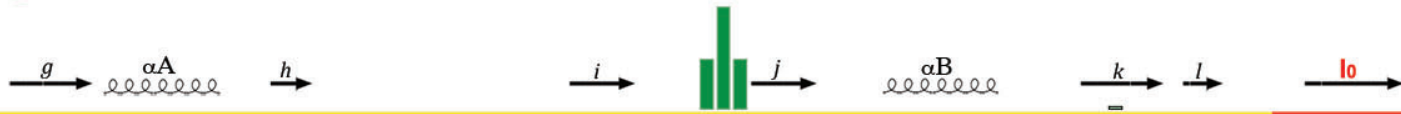

MEDKAWLVHRQWFLDLP LPWL PGADTQGS NWI QKE T LVTFKNP HAKKODDVVLGSQEGAMHTALTGATEI QMS SGNLLETGHLKCRLRMDKLQLKGMSYS

$\|_{250}$

260

270

$280^{\circ}$

290

300

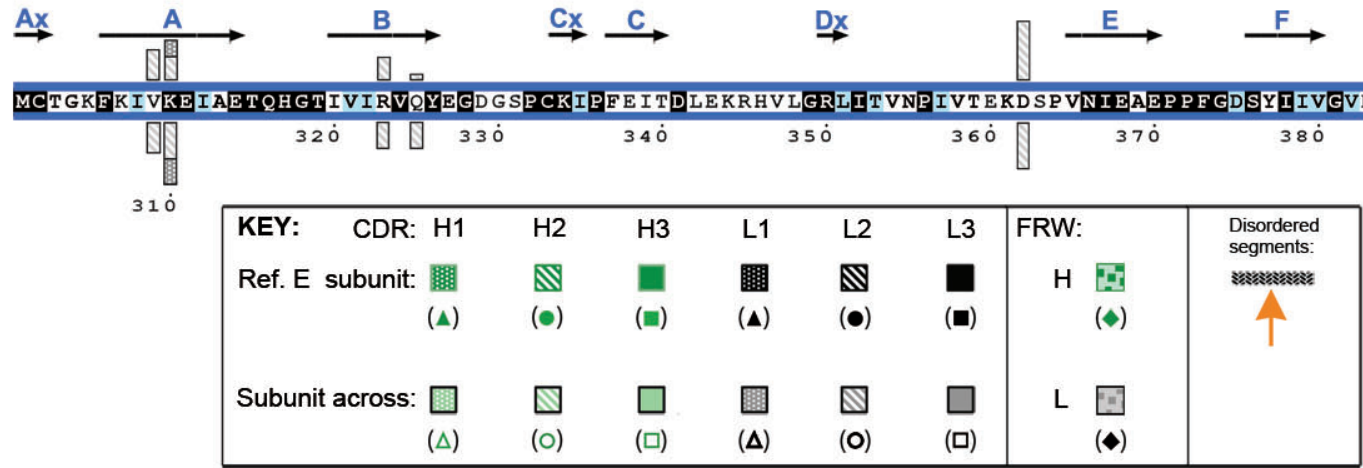

$\stackrel{\mathrm{G}}{\longrightarrow}$

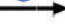




\section{d (continued) - DENV-2 sE / bnAB EDE1 ScFvC10 Complex}

III - 3D structure diagram with localization of epitopes

(second complex in the asymmetric unit of the crystal)

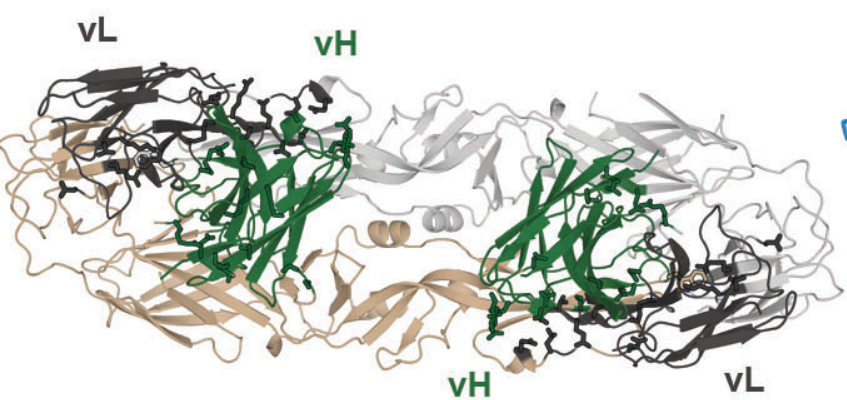
DEN2_FGA02 epitope D

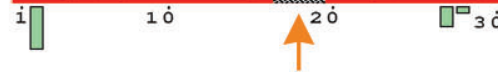

pitope C DEN2_FGA02 epitope D<smiles></smiles>

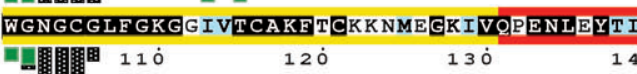

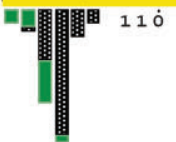

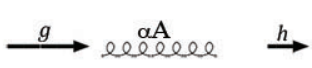

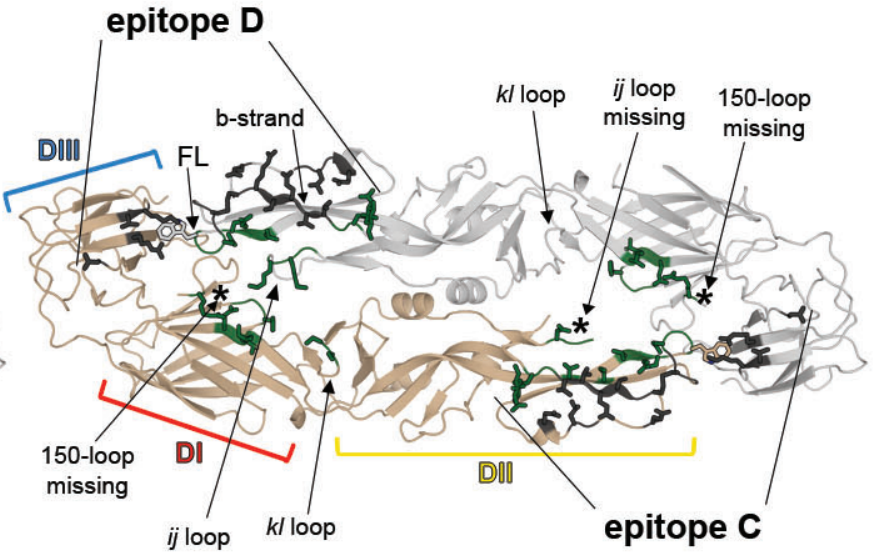

epitope C

IV - Histogram of number of atomic contacts of the bnAb per $\mathrm{SE}$ residue on epitopes $\mathrm{C}$ and $\mathrm{D}$

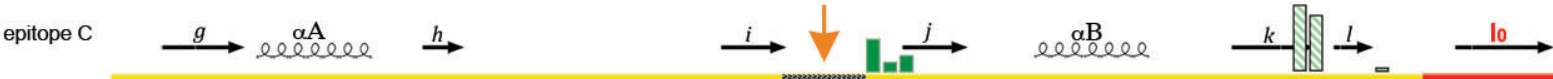

DEN2_FGA02 MEDKAWLVHRQWELDLP LPWLPGADTQGSNWI QKE TLVTFKNPHAKKODVVVLGSOEGAMHTALTGATEIQMS SGNL LETGHLKCRLRMDKLQLKGMSYS

epitope D

$210^{\circ} 220^{\circ} 230^{\circ}$

250

epitope C $\stackrel{A}{-}$

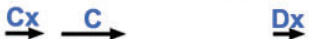

$\stackrel{\mathrm{F}_{0}}{\longrightarrow} \quad \downarrow \stackrel{\mathrm{G}_{0}}{\longrightarrow} \stackrel{\mathrm{H}_{0}}{\longrightarrow}$

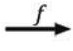

DEN2_FGA02 MCTGKEKIVKEIAETQHGTIVIRVQYEGD GSPCKIPFEITDLEKRHVLGRLITVNPIVTEKD SPVNIEAEP PFGDSYIIVGVEPGQLKLNWFKKG

epitope D

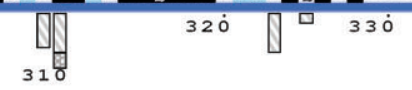

$340^{\circ}$

$4^{350}$

$360^{\circ} 370^{\circ}$

$380^{\circ}$

390

\begin{tabular}{|c|c|c|c|c|c|c|c|}
\hline $\begin{array}{l}\text { KEY: CDR: H1 } \\
\text { Ref. E subunit: 聞 } \\
\end{array}$ & $\begin{array}{l}\mathrm{H} 2 \\
\mathbb{N} \\
\text { (•) }\end{array}$ & $\begin{array}{l}\mathrm{H3} \\
\text { (回) }\end{array}$ & 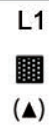 & $\begin{array}{l}\mathrm{L} 2 \\
\mathbb{N} \\
(\bullet)\end{array}$ & (a) & $\begin{aligned} \text { FRW: } & \\
\mathrm{H} & \text { (४) }\end{aligned}$ & $\begin{array}{l}\text { Disordered } \\
\text { segments: } \\
\text { sazszassa }\end{array}$ \\
\hline $\begin{array}{l}\text { Subunit across: } \square \\
(\Delta)\end{array}$ & $\underset{(0)}{\mathbb{Q}}$ & $\begin{array}{l}\square \\
(\square)\end{array}$ & $\begin{array}{l}\text { 罒 } \\
(\Delta)\end{array}$ & $\underset{(0)}{\mathbb{Q}}$ & $\begin{array}{l}\square \\
(\square)\end{array}$ & L & \\
\hline
\end{tabular}




\section{Histograms of $E$ contacts per antibody residue:}

The following pages mirror the diagrams displayed above, this time showing the number of contacts per antibody residue along the amino acid sequence of the bnAbs.

In each page, Part I shows the bnAb variable domain extracted from the corresponding complex, colored grey (VH dark grey, VL light grey) with somatic mutations in red and junction residues arising from recombination in green. Side chains involved in contacts are displayed in ball and stick and labeled. Part II shows the histogram of the number of atomic contacts per residue, color-coded according to the key to indicate the region of $\mathrm{sE}$ that is contacted (in parenthesis, the symbol used in ED Fig. $4 \mathrm{~b}$ to mark the corresponding contact). The sequence numbering and the background corresponds to Kabat convention (as in ED Fig. 4b). The CDRs corresponding to the IMGT convention are displayed as dotted orange lines above the sequences. Somatic mutations are in red, residues arising from VDJ (or VJ) recombination are in green. 
a bnAb EDE2 A11 residues in contact with DENV-2 sE dimer

I - 3D diagram

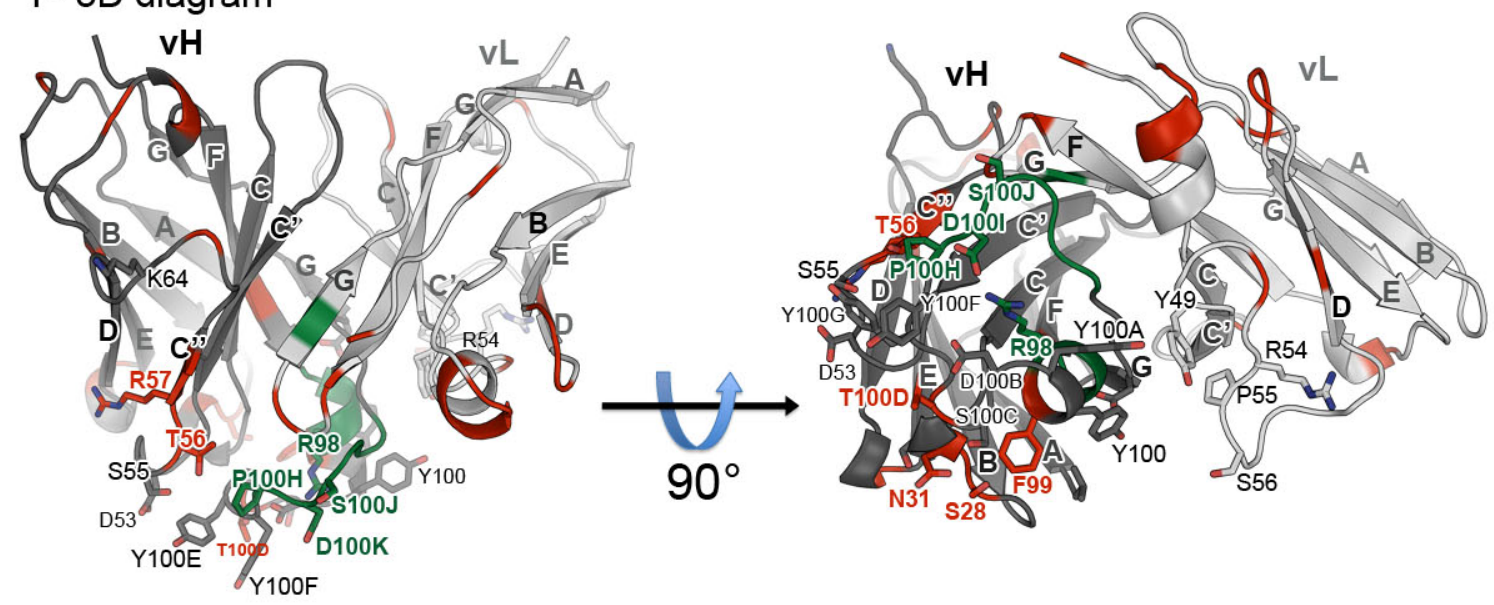

II - Linear diagram with histogram of contacts

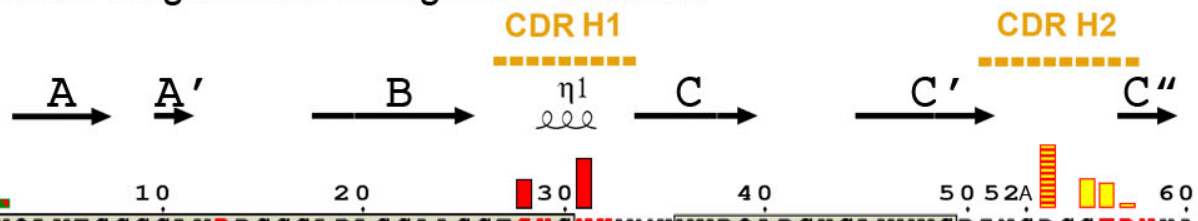

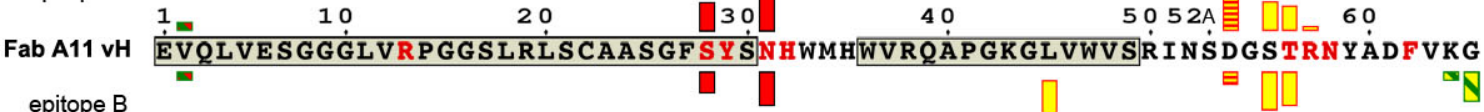

epitope A epitope B

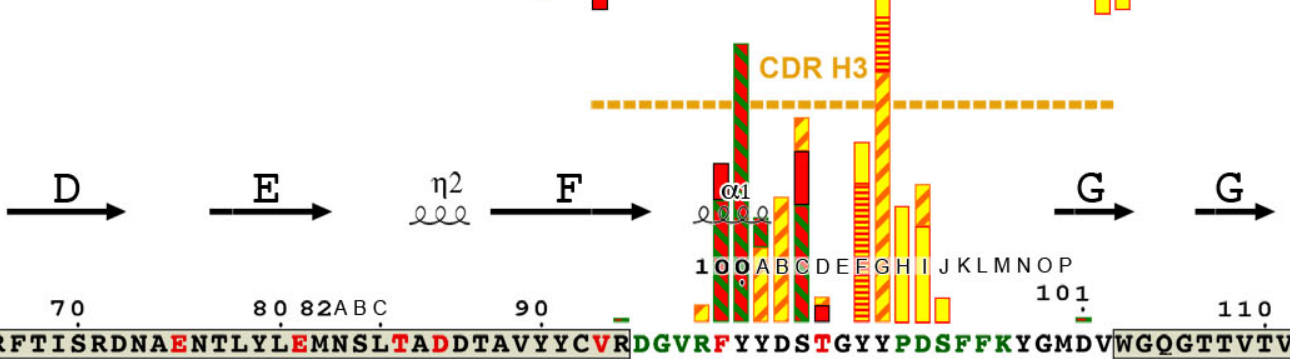

epitope A Fab A11 vH RFTISRDNAENTLYLEMNSLTADDTAVYYCVRDGVRFYYDSTGYYPDSFFKYGMDVWGQGTTVTV epitope B

Fab A11 VL Q்SVLTQPVSVंSGSPGQSITISCTGTSSNÄDTYNLVSWYQRPGKAPKLMIYEGTK epitope B
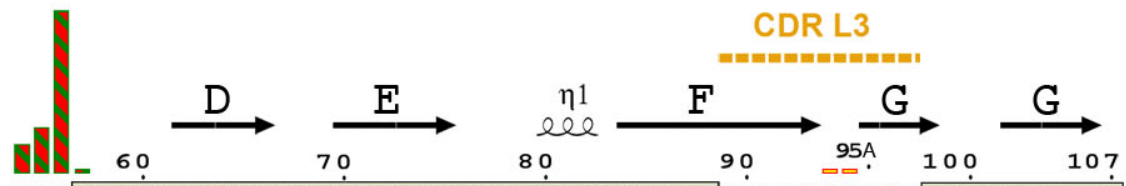

Fab A11 vL RPSGVSNRFSASKSATÁASLTISGLQPEDEADYYCCS YATSRTLVFGGGTKLTVV epitope B
KEY

( $\square$ ) $b$ strand

$(\triangle)$ N67 glycan

$(\diamond)$ Fusion loop

(○) $i j$ loop

$(\square)$ DII across

(घ) DI across

(৯) N153 glycan (across)

(घ) DIII across 
b bnAb EDE2 B7 residues in contact with DENV-2 sE dimer I - 3D diagram
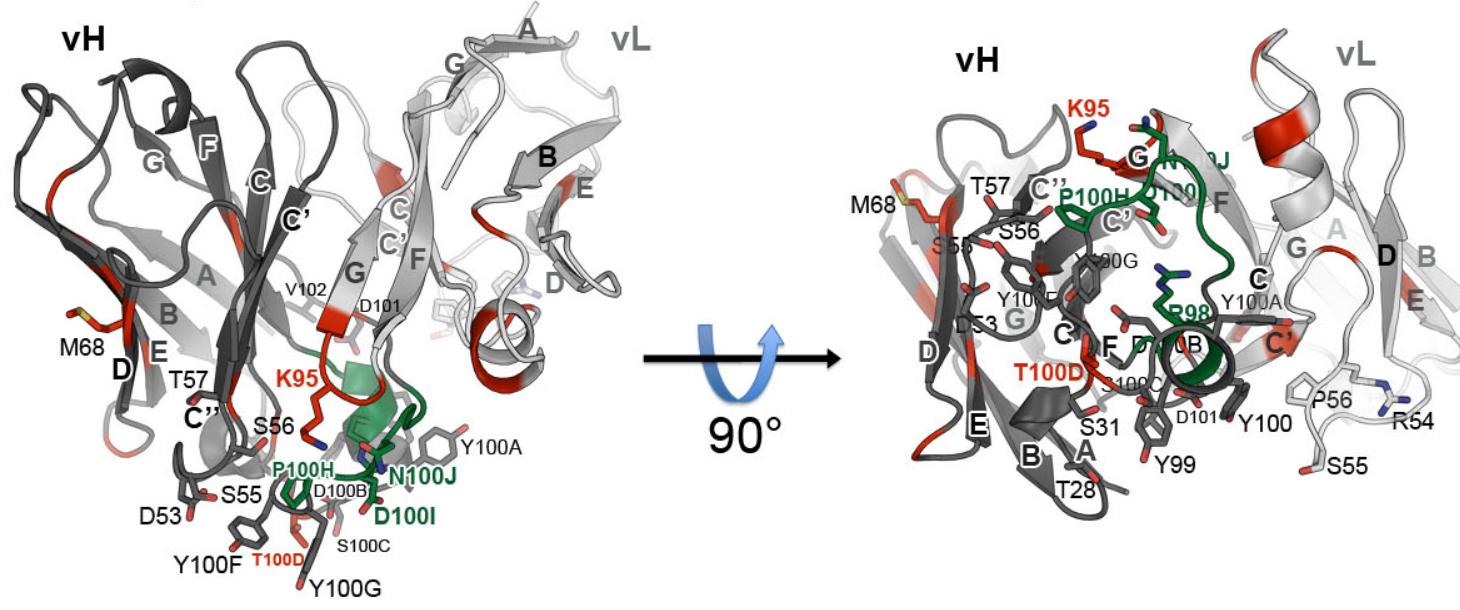

II - Linear diagram with histogram of contacts

CDR H1

\section{CDR H2}

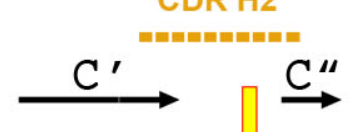

Fab B7 vH epitope B

epitope A Fab B7 vH

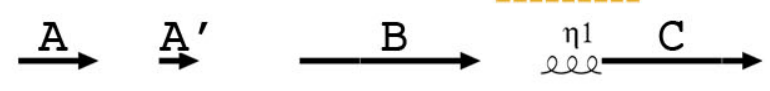
$\begin{array}{cccc}1 & 10 & 20 & 40\end{array}$

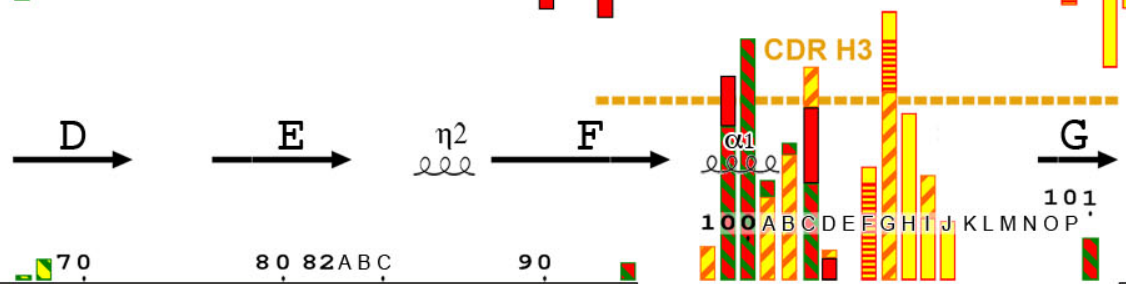

110

RFMISRDNSKNTVYLHMNGLRAEDTAVYFCARDGVRYYYDSTGYYPDNFFOYGLDVWGQGTTVTV epitope B
CDR L1

111
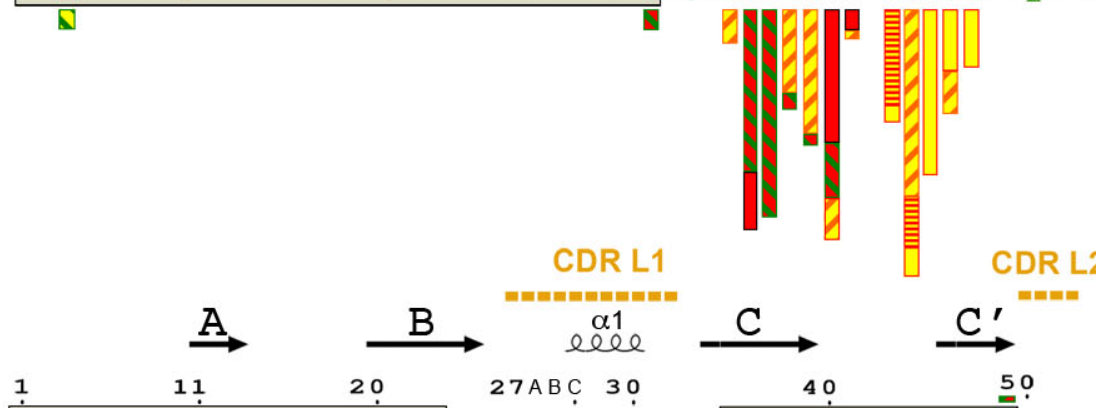

CDR L2

Fab B7 vL QंSALTQPASVंSGSPGQSITISCTGISSDVETYNLVSWYEQHPGKAPKLIIY EASK

epitope B

epitope A Fab B7 vL RPS GVSNRF SGSKSGNTASLAISGLQAंEDEADYYCC Sं YAGGKS LVFGGंGTRLTVL epitope B
KEY

( $) b$ strand

- $\diamond$

(घ) N67 glycan

-

(ム) Fusion loop

( ) ij loop

( ) DIl across

( ) DI across

( ) N153 glycan (across)

( ) DIII across 
d bnAb EDE1 C10 residues in contact with DENV-2 sE dimer

I - 3D diagram
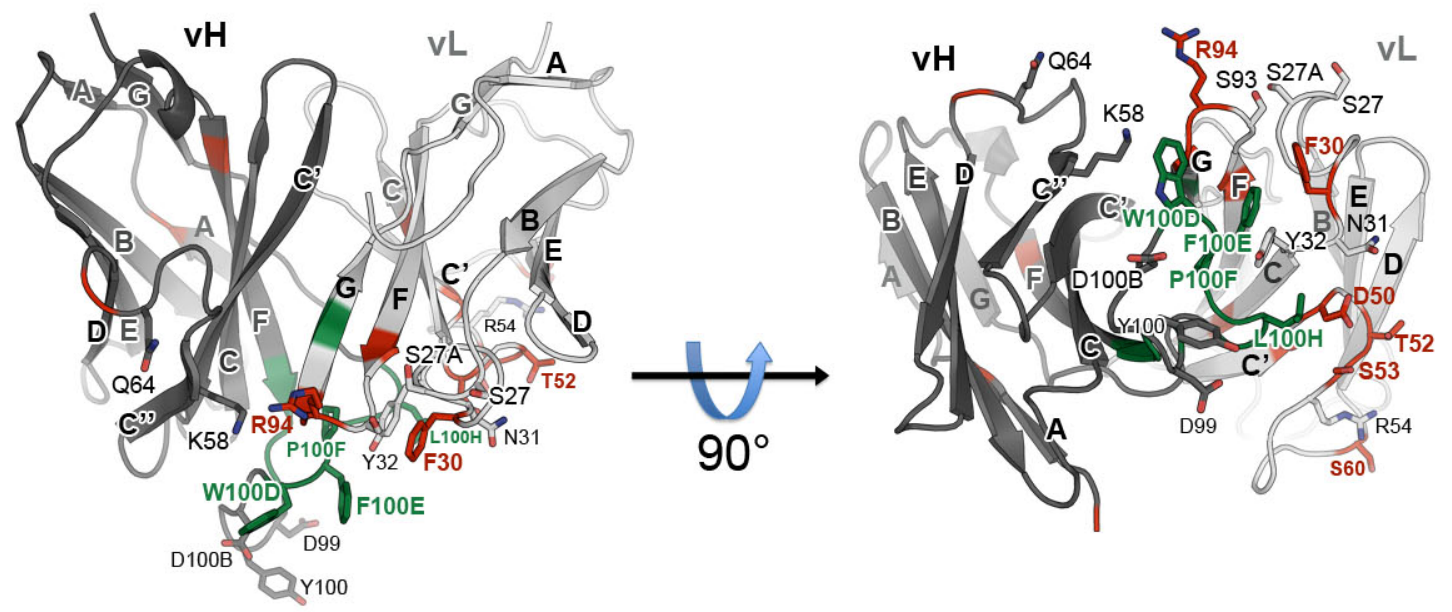

II - Linear diagram with histogram of contacts

\section{CDR H1}

CDR H2

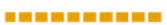

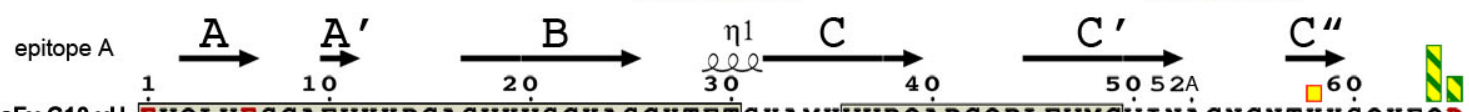
SCFV C10 vH epitope B

epitope B

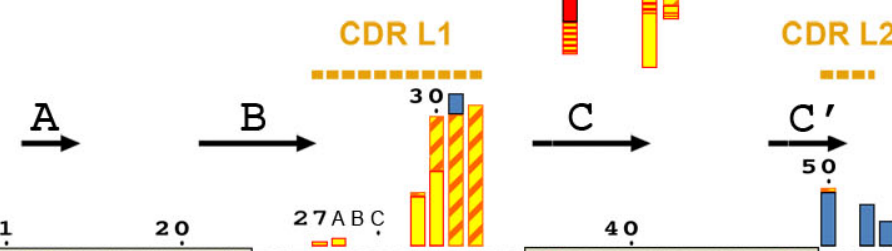

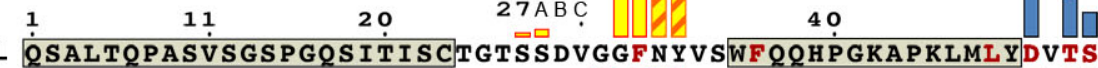

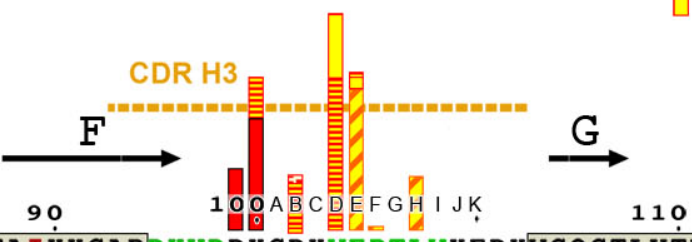

scFv C10 vL QSALTQPASVंS SS PGQS I T ISCTGTS D D VGFNY
epitope B

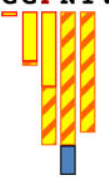

epitope A

epitope A
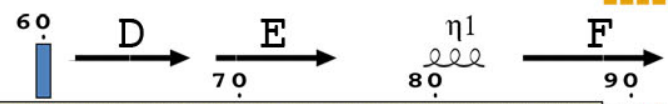

\section{CDR L3}

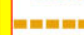
SCFv C10 VL RPSGVSSRF SGSKSGNTASLT ISGLQAEDEADY YCSSHTSRGTWVFGGGTKLTVL epitope B П
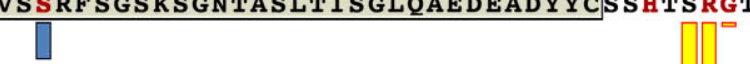

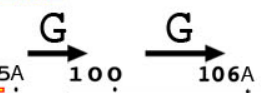

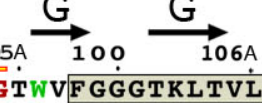

KEY

( $\square$ ) $b$ strand

$(\triangle)$ N67 glycan

$(\diamond)$ Fusion loop

(○) ij loop

(घ) DIl across

(घ) DI across (A) N153 glycan
(across)

(घ) DIII across 
d (continued) bnAb EDE1 C10 residues in contact with DENV-2 sE dimer (second complex in the asymmetric unit of the crystal)

I - 3D diagram
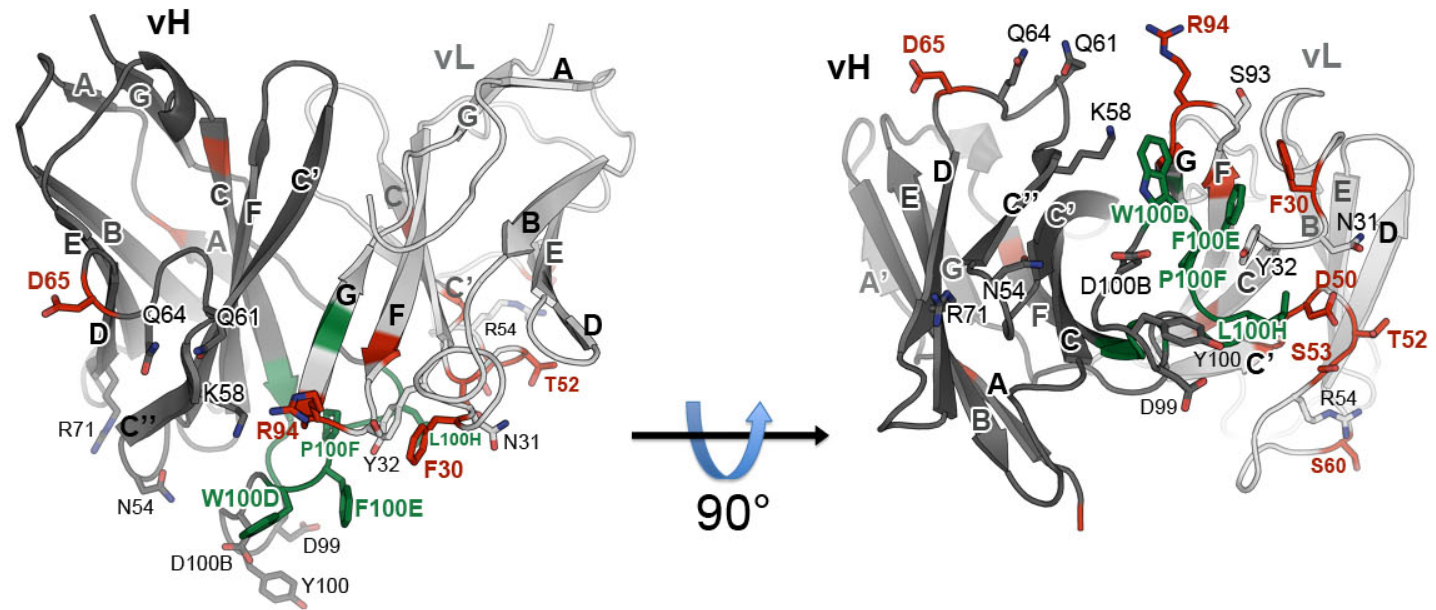

II - Linear diagram with histogram of contacts

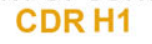

CDR H2

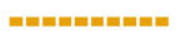

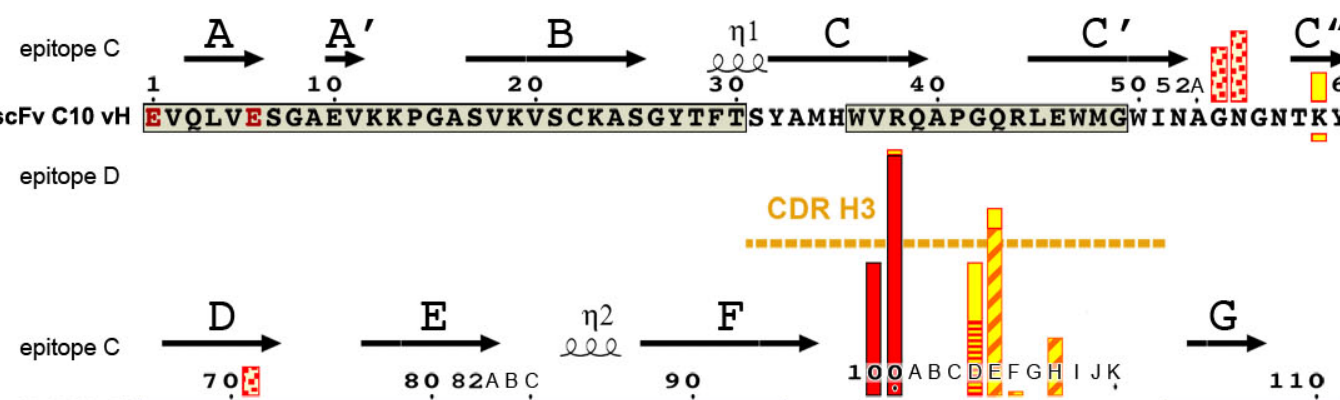

ScFV C10 vH RVTI ITRDTSASTA YMEELSSLRSEDTAIYYCARD KVDDYGDY WFPTLWYFD YWGQGTLVTV

epitope D

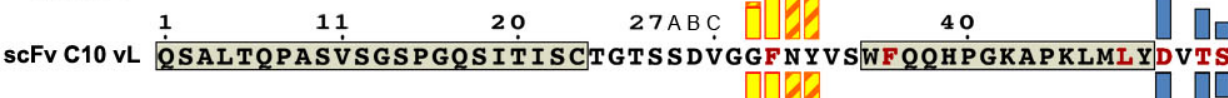

epitope C

CDR L1

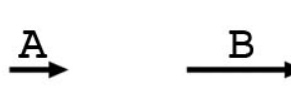

scF epitope D

epitope C

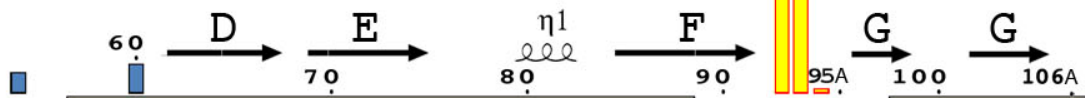
SCFV C10 vL RP SGVSSRF SGS KSGNTASLT I SGLQAEDEADY YCS SHTSRGTWVFGGGTKLTVL epitope D 